\title{
THE
}

$1-8-1998$

\section{A Semi-Empirical Potential for Simulations of Transition Metal Clusters: Minima and Isomers of $\mathrm{Nin}_{\mathrm{n}}(\mathrm{n}=2-13)$ and their Hydrides}

E. Curotto

University of Rhode Island

A. Matro

University of Rhode Island

David L. Freeman

University of Rhode Island, dfreeman@uri.edu

J. D. Doll

Follow this and additional works at: https://digitalcommons.uri.edu/chm_facpubs

Terms of Use

All rights reserved under copyright.

\section{Citation/Publisher Attribution}

Curotto, E., Matro, A., Freeman, D. L., \& Doll, J. D. (1998). A Semi-Empirical Potential for Stimulations of Transition Metal Clusters: Minima and Isomer of $\mathrm{Ni}_{n}(\mathrm{n}=2-13)$ and Their Hydrides. Journal of Chemical Physics, 108(2), 729-742. doi: 10.1063/1.475433

Available at: http://dx.doi.org/10.1063/1.475433

This Article is brought to you for free and open access by the Chemistry at DigitalCommons@URI. It has been accepted for inclusion in Chemistry Faculty Publications by an authorized administrator of DigitalCommons@URI. For more information, please contact digitalcommons-group@uri.edu. 


\title{
A semi-empirical potential for simulations of transition metal clusters: Minima and isomers of $\mathrm{Ni}_{n}(n=2-13)$ and their hydrides
}

\author{
E. Curotto, Alexander Matro, ${ }^{\text {a) }}$ and David L. Freeman \\ Department of Chemistry, University of Rhode Island, Kingston, Rhode Island 02881 \\ J. D. Doll \\ Department of Chemistry, Brown University, Providence, Rhode Island 02912
}

(Received 28 July 1997; accepted 30 September 1997)

\begin{abstract}
A potential energy surface (PES) for bare, mono and di-hydrogenated nickel clusters is constructed using the extended-Hückel approximation. The parameters are optimized and good agreement with theoretical and experimental results is obtained without including a posteriori coordination dependent terms. The global minimum and the first few low-lying isomers of several nickel clusters are investigated using a variety of minimization techniques. The difference in energy between isomers is much smaller than the Ni-Ni dissociation energy. Both geometric and optical isomers are found for many cluster sizes. In some cases symmetric nuclear configurations give rise to orbital degeneracies in the adiabatic surface which lead to distortions. The hydrogen atom is most frequently found on the surface. All isomers of $\mathrm{Ni}_{n} \mathrm{H}_{2}$ contain a dissociated hydrogen molecule. The results are in good agreement with quantitative and qualitative experimental findings on this system.

(C) 1998 American Institute of Physics. [S0021-9606(98)00702-8]
\end{abstract}

\section{INTRODUCTION}

If $a b$ initio potential energy surfaces could be evaluated with sufficient speed for many-particle systems, the determination of static, dynamic and thermodynamic properties would be possible within statistical uncertainties and the Born-Oppenheimer approximation. While much progress has been made in recent years to enable the determination of the potential energy during numerical simulations, ${ }^{1}$ the cost in computer time can range from high to prohibitive. For the case of transition metal clusters of interest to the current study, $a b$ initio simulations represent a future goal rather than a current reality. As an alternative quantum chemical approach, semi-empirical methods ${ }^{2}$ are a useful approximation to full $a b$ initio calculations ${ }^{3,4}$ when used to obtain potential surfaces for transition metal clusters. Semi-empirical methods provide a fast and simple way to reproduce approximately the quantum mechanical forces that determine the physical properties of the interacting system. These forces require a quantum description owing to the strong directional character of the $d$ and $p$ orbitals. The $d$ bands can play a determining role in the bonding between transition metal atoms. In the semi-empirical method the computational effort is diminished by a parameterization of the interaction matrix between the $s, p$ and $d$ atomic basis. With these models it is possible to carry out dynamical and statistical simulations with reasonable amounts of computational resources. For transition metal clusters, most simulations ${ }^{5-7}$ to date have used less expensive potential functions. Examples include the embedded atom model (EAM $)^{5-7}$ and the corrected effective medium theory (CEM) ${ }^{8,9}$ In the embedded atom model $^{5}$ the overall potential energy is constructed from spherically symmetric charge distributions about each atom.

${ }^{\text {a)} P r e s e n t ~ a d d r e s s: ~} 302$ Osprey Ln, Voorhees, NJ 08043
The corrected effective medium theory is based on an approach similar in spirit to EAM. Previous studies of nickel clusters have used both these methods. ${ }^{5-9}$ While semiempirical methods provide potentials that are by no means exact, physical effects characteristic of a class of systems are observable that cannot be seen with alternate representations of the interatomic potential. Examples of these effects are discussed in the current work.

A popular set of semi-empirical approaches that have been used for transition metals are the tight binding methods. An example is the extended-Hückel approximation ${ }^{10}(\mathrm{EH})$ that has been applied to a range of systems including covalent organic molecules and transition metal compounds. ${ }^{11}$ The EH approach has been successfully applied to transition metal clusters by Anderson, ${ }^{12}$ who studied several small systems of up to six atoms, of $\mathrm{Ti}, \mathrm{Cr}, \mathrm{Fe}$ and $\mathrm{Ni}$. Another variant of the Hückel approximation has been developed by van Schilfgaarde and Harrison ${ }^{13-15}$ and the resulting methodology has been applied to study of semiconductor clusters as well as bulk semiconductors and transition metals by a number of authors. This semi-empirical technique differs from the EH approximation mainly in the way the off diagonal elements of the Hamiltonian are approximated. We refer to this technique as modified tight binding scheme (MTB). A small sample of recently published applications of MTB to semiconductors is found in Refs. 16-20. MTB potentials have also been applied to transition metal clusters by a number of authors. In a series of publications Lathiotakis et al. ${ }^{21-24}$ describe a MTB potential energy surface model for nickel clusters. The resulting potential surface has been used to obtain structural information for a large number of Ni clusters.

As has been understood for some time, semi-empirical methods often underestimate the total binding energy of the global minimum of a semiconductor or transition metal clus- 
ter. This underestimate has been attributed to the method's neglect of free to hybrid atom promotional energies, ${ }^{19}$ and to the incorrect assumption made that the basis set overlap integrals are those at the bulk atom-atom separation. ${ }^{17}$ In fact, the average bond length for a metal or semiconductor cluster can be quite different from the bulk value. To correct the binding energy deficiency in the MTB scheme Tomańek and Schluter ${ }^{20}$ have proposed the addition of a simple bonddependent term $U_{\text {bond }}$ to the total binding energy of a series of minimized structures. This method is still used in many applications of semi-empirical theories to semiconductors and transition metals. This bond-counting term is awkward to use directly in dynamical simulations even if a smooth cutoff function is used for the determination of the number of bonds. ${ }^{16,18,19,22,23}$ In practice $U_{\text {bond }}$ has not been used to derive forces and has only been added to the minimized geometries after dynamical simulations have been completed. A goal of the current work is to construct a semi-empirical potential function where all the energetic contributions can be included everywhere on the potential surface.

In this paper we describe an improved semi-empirical method based on the EH approximation for Ni clusters. Our main improvement is a way to eliminate $U_{\text {bond }}$ altogether. We are encouraged in this process by the success of Ordejón, Lebedenko, and Menon ${ }^{17}$ who have been able to eliminate $U_{\text {bond }}$ in semiconductor clusters by re-introducing the full dependence of the overlap between adjacent atoms.

In the current work we develop a semi-empirical potential energy function for nickel clusters that as closely as possible satisfy the goals that follow:

(1) Obtain a potential energy model which efficiently reproduces the strong directional nature of the $p$ and $d$ orbitals in transition metal bonding and hence the quantum nature of the electronic Born-Oppenheimer surface.

(2) Eliminate the need for any artificial term dependent on coordination to achieve agreement between the EH approximation and $a b$ initio calculations, experimental cohesive energies and empirical structural information.

(3) Parameterize the model potential for nickel clusters and use this to obtain structural and energetic information about the global minima of several isomers for a range of sizes.

(4) Generate a model potential that is useful for future classical, quantum and statistical simulations from which important dynamic and thermal properties of the clusters can be obtained.

The contents of the remainder of this paper are organized as follows: Section II contains a brief outline of the extended-Hückel method, a description of the method used to obtain the best set of parameters for the EH matrix, and a description of the methods we use to find minima and isomers of the potential energy surface. In Section III we discuss the results of searches for the minima of clusters from $\mathrm{Ni}_{2}$ to $\mathrm{Ni}_{14}, \mathrm{Ni}_{n} \mathrm{H}$ and $\mathrm{Ni}_{n} \mathrm{H}_{2}$. Finally, in Section IV we summarize and discuss our results.

\section{METHOD}

\section{A. Basic theory}

It is known that the standard EH approximation requires an additional repulsive term in order to reproduce a realistic potential. The overall potential energy is calculated by a sum of two terms,

$$
U_{\text {tot }}=U_{\text {rep }}+U_{\text {eh }} \text {, }
$$

where $U_{\text {rep }}$ is a sum of pair-wise repulsion terms between $N$ atoms

$$
U_{\text {rep }}=\sum_{i}^{N} \sum_{j>i}^{N} \chi_{i, j} .
$$

Several approaches have been proposed to represent the repulsive potential $\chi_{i, j}$. Among these we note the Anderson Hoffmann approach ${ }^{25}$ based on charge density functionals, and the squared overlap method of Lee. ${ }^{26}$ We have chosen a simpler exponential scaling function with respect to the atom-atom distance $r_{i, j}$ which is commonly used in MTB calculations,

$$
\chi_{i, j}=\chi_{0} \exp \left[-\alpha\left(\frac{r_{i, j}-d}{d}\right)\right] .
$$

Following Lathiotakis et al. ${ }^{21}$ we have set $d$ to the $\mathrm{Ni}-\mathrm{Ni}$ bulk value separation, $d=2.498 \AA$.

The electronic energy is a sum over occupied EH electronic energy levels

$$
U_{\mathrm{eh}}=\sum_{n} b_{n} E_{n},
$$

where $b_{n}$ is the occupancy number for a given molecular orbital and the set $\left\{E_{n}\right\}$ is the solution to the generalized eigenvalue problem

$$
\mathbf{C}_{n}^{\dagger}\left(\mathbf{H}-E_{n} \mathbf{S}\right) \mathbf{C}_{n}=0 .
$$

First-principle classical or Brownian simulations, used in this work, are carried out by integrating Newton's equations of motion

$$
m \frac{\partial^{2} \mathbf{x}}{\partial t^{2}}=-\frac{\partial U_{\text {tot }}}{\partial \mathbf{x}}-\gamma \frac{\partial \mathbf{x}}{\partial t}+F(T, t),
$$

where $\mathbf{x}$ is a vector containing the Cartesian coordinates of the nuclei, $\gamma$ is the coefficient of friction and $F(T, t)$ is a random force ${ }^{6}$ with correlation function given by the standard expression for white noise at temperature $T$. The derivative with respect to $x$ of the repulsive part of $U_{\text {tot }}$ is trivially obtained from Eq. (3). The derivative of the electronic part is obtained by using the Hellmann-Feynman theorem. From Eq. (5) we obtain ${ }^{19}$

$$
\frac{\partial E_{n}}{\partial x_{i}}=\mathbf{C}_{n}^{\dagger}\left(\frac{\partial \mathbf{H}}{\partial x_{i}}-E_{n} \frac{\partial \mathbf{S}}{\partial x_{i}}\right) \mathbf{C}_{n} .
$$

The generalized eigenvalue problem of Eq. (5) is solved by applying the standard Cholesky decomposition of the overlap matrix $\mathbf{S}$ 


$$
\mathbf{S}=\mathbf{L L}^{T},
$$

where $\mathbf{L}$ is a lower triangular matrix.

\section{B. Parameterization of the EH matrix}

In standard EH theory, a direct proportionality between the overlap element $S_{i, j}$ and the coupling Hamiltonian element $H_{i, j}$ is assumed

$$
H_{i, j}=K\left(\frac{H_{i, i}+H_{j, j}}{2}\right) S_{i, j},
$$

whereas the diagonal element $H_{i, i}$ is set to the experimental ionization energy of the atomic orbital $i, H_{i, i}=\epsilon_{n l}$. Consistent with the ordering of the ionization energies of this particular parameter set, the ground state of $\mathrm{Ni}$ is $4 s^{2} 3 d^{8}$. This ground state configuration is the same that has been used by Anderson. ${ }^{12}$ For nickel, the atomic basis set consists of a $4 s$, three $4 p$ and five $3 d$ Slater-Koster ${ }^{27}$ orbitals for each atom. We use double-zeta representation for the $d$ orbitals. This gives a set of 12 parameters to be determined empirically; the three on-site ionization energies $\left(\epsilon_{4 s}, \epsilon_{4 p}, \epsilon_{3 d}\right)$, the four orbital exponents $\left(\zeta_{4 s}, \zeta_{4 p}, \zeta_{3 d 1}, \zeta_{3 d 2}\right)$, the double-zeta normalizing coefficients $\left(c_{1}\right.$ and $\left.c_{2}\right)$, the two parameters for the repulsive energy ( $\alpha$ and $\chi_{0}$ ), and the Hückel parameter $K$.

The atomic parameters $\zeta_{n l}$ used by Anderson ${ }^{12}$ are obtained by fitting to accurate atomic Hartree-Fock calculations. We have carried out a preliminary search for the cluster minima using Anderson $^{12}$ parameters. We have discovered, however, that the resulting potential model underestimates the binding energy of clusters by several eV. Furthermore, the binding energy difference between global minima of adjacent sizes do not follow the experimental trend. ${ }^{28}$ These experimental findings were not available at the time that Anderson carried out his investigation. In light of these preliminary minimization results we optimize the scaling with distance of all the overlaps (i.e., the parameters of the atomic basis set $\zeta_{n l}$ ). This achieves better agreement between EH, $a b$ initio and experimental binding energies without making use of any bond counting term. The main criteria used to optimize the parameters are the following:

(1) The potential should accurately reproduce binding energies obtained from $a b$ initio calculations performed on structures of many different sizes.

(2) The model should reproduce the experimental trend in the cohesive energies of minimized structures in the entire range under study.

(3) The model should predict structures for the global minima that are in qualitative agreement with the nitrogen adsorption experiments of Parks et al. ${ }^{29}$

The on-site ionization energies used by Anderson ${ }^{12}$ are experimental values except for $\epsilon_{3 d}$. He noted that it is necessary to increase this energy from -10.0 to $-7.7 \mathrm{eV}$ and raise $\zeta_{4 s}$ by $0.2 \mathrm{bohr}^{-1}$ in order to obtain binding for the dimer. We use the same on-site energies that Anderson used. The optimization of the remaining parameters is carried out in two stages. First we perform an initial adjustment of these by fitting to $a b$ initio calculations on $\mathrm{Ni}_{4}$ performed by Mly- narski and Salahub ${ }^{30}$ and $\mathrm{Ni}_{6}, \mathrm{Ni}_{8}, \mathrm{Ni}_{19}$ by Rösch and Ackermann. ${ }^{31}$ In these ab initio studies, each cluster is minimized by imposing symmetry constraints. The authors report the binding energies of the geometries at two different atomatom separations. In addition, the experimental ${ }^{32}$ binding energy $-2.068 \mathrm{eV}$, the bond length $2.20 \AA$ and the frequency $330 \mathrm{~cm}^{-1}$ of the dimer ${ }^{21}$ are included in the data pool. The fitting is achieved by minimizing the sum of the squares of the percent deviation between EH and $a b$ initio binding energies. The four Slater exponents $\zeta_{n l}$ and the two double-zeta coefficients, $c_{1}$ and $c_{2}$ are obtained by a random walk. For each set of $\zeta_{n l}$ and $c_{i}$ obtained randomly, $\alpha$ and $\chi_{0}$ and the Hückel parameter $K$ are systematically varied until the dimer properties are accurately reproduced. This procedure is necessary to eliminate much of the correlation that exists among the nine parameters. ${ }^{19}$ The only notable change in parameters is a relatively large increase in $\zeta_{4 s}$ to three and half times the value used by Anderson. ${ }^{12}$ A further small adjustment of all the parameters is carried out to assure that clusters larger than $\mathrm{Ni}_{7}$ favor structures with high coordination. Global minima geometries of clusters larger that $\mathrm{Ni}_{7}$ are quite sensitive to the values chosen for the parameters, especially those describing the $d$ basis.

The only notable quantitative discrepancies which we are unable to resolve are the cohesive energies of $\mathrm{Ni}_{3}$ and $\mathrm{Ni}_{4}$ which we overestimate by approximately $2 \mathrm{eV}$ and the vibrational frequency of $\mathrm{Ni}_{2}$ (see Table II and Fig. 6). Curiously, many other potential models applied to this system seem to show the trend for $\mathrm{Ni}_{3}$ and $\mathrm{Ni}_{4}$ reported here rather than the experimental trend (see Fig. 6 and the associated discussion). We have made many attempts to find a set of parameters that reproduces the sequential cohesive energies of the clusters, while at the same time gives a smaller vibrational frequency of the dimer and a value for $\zeta_{4 s}$ closer to previously reported exponents. We also have tried to vary the on-site energies together with all the other parameters. We have been, however, unable to find a set of parameters that simultaneously satisfies all these criteria. The reproduction of all these properties simultaneously may be beyond the capability of the simple electronic model we are implementing. Furthermore, the values of the orbital parameters optimized in the manner described above need not be in agreement with values obtained from accurate variational calculations using first principle repulsive forces. As such, these parameters may not be transferable to other applications of extended-Hückel theory.

In Table I we report the basis sets and repulsive parameters. Where possible the optimized values are compared with those used by Anderson. ${ }^{12}$ The repulsion parameters $\alpha_{\mathrm{Ni}-\mathrm{Ni}}$ and $\chi_{\mathrm{Ni}-\mathrm{Ni}}$ reported in the column labeled Ref. 12, are the values optimized when the $\zeta_{n l}$ 's and the $\epsilon_{n l}$ 's of Ref. 12 are used.

\section{The genetic algorithm}

We minimize the structures in a variety of ways, but we preferentially use Brownian dynamics simulated annealing [see Eq. (6)]. ${ }^{6}$ We use alternative methods such as Monte 
TABLE I. Optimized EH and repulsion parameters.

\begin{tabular}{ccc}
\hline \hline Parameter & Ref. 12 & Opt. \\
\hline$\epsilon_{1 s}$ & -13.606 & -13.606 \\
$\epsilon_{4 s}$ & -7.635 & -7.635 \\
$\epsilon_{4 p}$ & -3.99 & -3.99 \\
$\epsilon_{3 d}$ & -7.70 & -7.70 \\
$\zeta_{1 s}$ & 1.20 & 1.991 \\
$\zeta_{4 s}$ & 1.70 & 5.982 \\
$\zeta_{4 p}$ & 0.86 & 0.987 \\
$\zeta_{3 d 1}$ & 5.70 & 5.580 \\
$\zeta_{3 d 2}$ & 1.95 & 2.519 \\
$c_{1}$ & 0.5705 & 0.3974 \\
$c_{2}$ & 0.6317 & 0.7147 \\
$K_{\mathrm{Ni}-\mathrm{Ni}}$ & 1.4368 & 2.407 \\
$\alpha_{\mathrm{Ni}-\mathrm{Ni}}$ & 20.047 & 21.084 \\
$\chi_{\mathrm{Ni}-\mathrm{Ni}}$ & 0.0154 & 0.047 \\
$K_{\mathrm{Ni}-\mathrm{H}}$ & - & 3.035 \\
$\alpha_{\mathrm{Ni}-\mathrm{H}}$ & - & 9.521 \\
$\chi_{\mathrm{Ni}-\mathrm{H}}$ & - & 0.582 \\
$K_{\mathrm{H}-\mathrm{H}}$ & - & 2.603 \\
$\alpha_{\mathrm{H}-\mathrm{H}}$ & - & 1.750 \\
$\chi_{\mathrm{H}-\mathrm{H}}$ & - & 7.858 \\
\hline \hline
\end{tabular}

Carlo simulated annealing (MCSA), the Newton-Rapson, and the conjugate gradient method ${ }^{35}$ to double check the validity of our results. In particular MCSA requires no gradient evaluation and we use this technique extensively to test our programs.

To find the lowest energy structure for each cluster size and composition, simulated annealing methods require many trajectories begun from random configurations. The number of trajectories required can be reduced using genetic algorithmic methods. ${ }^{36-40}$ We find the genetic algorithm to be indispensable for structures with more than six atoms. The first generation of parents is obtained by minimizing a set of randomly generated structures. The starting Cartesian coordinates for these trajectories are uniformly drawn from a box of sufficiently large size. Like Zeiri ${ }^{39}$ we use the Cartesian coordinates of the nuclei as our control variables. The starting coordinates for the next generation of children is obtained by a single operation from two parent configurations. Both parents are cut by a randomly placed plane and rejoined to form the children. Our genetic operator is very similar to that employed by Deaven and $\mathrm{Ho}^{38}$ for Lennard-Jones clusters. The parents with lower energy are selected with higher probability according to the following normalized distribution,

$$
f_{i}=\frac{\exp \left(-V_{i}\right)}{\sum_{i=1}^{N_{\text {pop }}} \exp \left(-V_{i}\right)},
$$

where $N_{\text {pop }}$ is the total number of parents and $V_{i}$ is the binding energy of isomer $i$. We find that $N_{\text {pop }}=64$ works quite well for our purposes. $N_{\text {pop }}$ structures are generated at each pass. We use Brownian dynamics simulated annealing ${ }^{6}$ to quench the resulting children. Configurations are cooled to zero Kelvin abruptly in order to arrive at the closest local minimum.
After equivalent structures are removed in the manner we describe below, the remaining children are added to the parents and the result is sorted by energy; the lowest $N_{\text {pop }}$ configurations become the new parents for the following generation. Our search appears to be comprehensive up to $\mathrm{Ni}_{7}$ for bare clusters, up to $\mathrm{Ni}_{5} \mathrm{H}$ and up to $\mathrm{Ni}_{4} \mathrm{H}_{2}$ for the hydrogenated systems. For larger systems we update the parent configurations and keep the lowest 64 structures. The genetic algorithm is terminated when for two consecutive generations no new parent structure is found. This yields the lowest discovered candidate isomers for a given system. We find that between 1000 to 2000 trajectories (18-30 generations) are sufficient to achieve this termination criteria for clusters in the size range considered here.

After the lowest 64 candidates are found, these are further quenched so that the fractional change in energy between subsequent steps is less than one part in $10^{6}$ and in some case to one part in $10^{12}$. This procedure gives us confidence that the lowest minimum reported for each system is most probably the global minimum and that the lowest few isomers for each system are found.

To confirm that the Brownian algorithm yields distinct minima, each minimum obtained for $\mathrm{Ni}_{3}$ to $\mathrm{Ni}_{7}, \mathrm{Ni}_{2} \mathrm{H}$ to $\mathrm{Ni}_{5} \mathrm{H}$ and $\mathrm{NiH}_{2}$ to $\mathrm{Ni}_{4} \mathrm{H}_{2}$ is quenched to one part in $10^{12}$ in the energy and then submitted to a Monte Carlo simulated annealing program. Moves are made uniformly with a starting step of $10^{-5}$ a.u. to assure that the algorithm does not accidentally hop out of a local well. After 100 consecutive passes are rejected, the step size is cut in half. A step smaller than $10^{-8}$ a.u. is used as convergence criterion. The Monte Carlo algorithm converges very quickly for each minimum found by the Brownian annealing method for all tested systems. An inspection of the eigenvalues of the Hessian matrix,

$$
\frac{\partial^{2} U_{\text {tot }}}{\partial x_{i} \partial x_{j}},
$$

determined numerically, for each minimum is also carried out. We find that all the isomers have $3 N-6$ positive eigenfrequencies and therefore are not transition states or other higher order saddles.

\section{The structural comparison algorithm}

To maintain diversity in the gene pool, identical children are removed. Because the energy difference between isomers is small, it is difficult to resolve differences between isomers using energy criteria alone without having to minimize to very small tolerances. When the parent set has evolved for several generations toward the lowest set of structures, several additional passes may create as few as one new parent at a time, and the process can become quite wasteful. We find it more efficient, therefore, to reduce the tolerance and compare the minima both topologically and energetically, by attempting to superimpose each child-child and child-parent pair with a set of translation-rotation operations.

To understand the structural comparison algorithm we consider two clusters $A$ and $B$. We choose a single atom from each of $A$ and $B$ as the common origin. We define a set 
TABLE II. Dimer properties calculated vs experiment.

\begin{tabular}{lccccrr}
\hline \hline & \multicolumn{2}{c}{$D^{0}(\mathrm{eV})^{\mathrm{a}}$} & \multicolumn{2}{c}{$r_{e}(\mathrm{bohr})^{\mathrm{b}}$} & \multicolumn{2}{c}{$\omega_{e}\left(\mathrm{~cm}^{-1}\right)^{\mathrm{c}}$} \\
Dimer & cal. & exp. & cal. & exp. & cal. & exp. \\
\hline Ni-Ni & 2.1416 & 2.042 & 4.1939 & 4.07 & 545 & 280 \\
Ni-H & 2.7464 & 2.70 & 2.7840 & 2.788 & 1994 & 2003 \\
$\mathrm{H}-\mathrm{H}$ & 4.4330 & 4.4781 & 1.4080 & 1.4010 & 4561 & 4401 \\
\hline \hline
\end{tabular}

${ }^{a}$ Potential well depth.

${ }^{\mathrm{b}}$ Equilibrium bond length.

${ }^{\mathrm{c}}$ Harmonic frequency.

of translations which has at most $N$ elements, where $N$ is the number of atoms in $B$. The second and third atom determine a common $z$ axis and a common $x-z$ plane, respectively, for $A$ and $B$. From these, a set of $(N-1)(N-2)$ pair of Euler angles is obtained and with these we rotate $B$. This yields at most $N(N-1)(N-2)$ combined operations to compare the two structures. If the two structures are equal, then at least one of these operations renders the coordinate set of $A$ equal to that of $B$. If, however, the two are different then there is no operation in this set that renders this equality. For each of the $N(N-1)(N-2)$ attempts at superimposing structure $A$ and $B$ in a pair, the distance between atom $i$ in structure $A$ and atom $i$ in structure $B$ is measured for all atoms. If a translation and rotation is found for which all such distances are less than $\sigma=0.8$ bohr then the structures are assumed identical. The parameter $\sigma=0.8 \mathrm{bohr}$ is sufficiently small to distinguish the first three isomers of $\mathrm{Ni}_{7}$, which, as we discuss in the next section, have very similar geometries. We test the same comparison procedure in the genetic algorithm with a smaller value of $\sigma$ for $\mathrm{Ni}_{7}$ but we find no additional minima. The $\sigma$ parameter must be adjusted carefully. If $\sigma$ is too small, then the parent set is not sufficiently diversified on each pass, and upon further minimization too few isomers are obtained. If $\sigma$ is too large then isomers that are geometrically similar can be mistaken as equal and thrown away. With this comparison technique we have discovered the existence of numerous pairs of degenerate structures of $\mathrm{Ni}_{n}$ which are non-superimposable mirror images, i.e., optical isomers. This feature, however, is not restricted to transition metal clusters. The highest energy isomer of a 7-atom Lennard-Jones cluster, for example, is optically active, a fact that seems to have escaped previous notice. In fact we find that three of the distinct minima of $\mathrm{Ni}_{7}$ resemble the structure of the highest energy isomer of a 7-atom Lennard-Jones cluster (see Fig. 3) and have degenerate enantiomers.

\section{RESULTS}

\section{A. $\mathrm{Ni}_{2}, \mathrm{NiH}$ and $\mathrm{H}_{2}$}

In Table II, the values of the experimental binding energy, bond length and vibrational frequency of $\mathrm{Ni}_{2}$ are taken from Refs. 33 and 34, respectively. During the search of the best set of parameters we include experimental data for the additional two dimers that are relevant to the current study, namely $\mathrm{NiH}$ and $\mathrm{H}_{2}$. Numerous investigations of nickel hydride have been published. We make use of experimental values cited in a recent theoretical study ${ }^{41}$ and in an earlier
TABLE III. Potential energy, average nearest-neighbor distance and number of bonds [see Eqs. (12) and (13) in the text] for bare nickel clusters.

\begin{tabular}{lccrcccc}
\hline \hline N.m & $\mathrm{V}(\mathrm{eV})$ & $\left\langle r_{e}\right\rangle$ (bohr) & $N_{b}$ & $\mathrm{~N} . \mathrm{m}$ & $\mathrm{V}(\mathrm{eV})$ & $\left\langle r_{e}\right\rangle$ (bohr) & $N_{b}$ \\
\hline 2.1 & -2.1416 & 4.1939 & 1 & 7.5 & -17.3064 & 4.4362 & 14 \\
3.1 & -5.2553 & 4.3042 & 3 & 7.6 & -17.2870 & 4.4451 & 13 \\
4.1 & -8.9862 & 4.3688 & 6 & 7.7 & -17.2837 & 4.5654 & 15 \\
5.1 & -11.9723 & 4.4018 & 9 & 7.8 & -17.2619 & 4.5045 & 15 \\
5.2 & -11.7449 & 4.4027 & 8 & 7.9 & -17.2233 & 4.4722 & 15 \\
5.3 & -10.8726 & 4.3432 & 7 & 7.10 & -17.1913 & 4.4753 & 15 \\
6.1 & -14.8420 & 4.4606 & 12 & 7.11 & -17.1886 & 4.4971 & 16 \\
6.2 & -14.8401 & 4.4560 & 12 & 7.12 & -17.1398 & 4.5218 & 16 \\
6.3 & -14.4742 & 4.4408 & 12 & 7.13 & -16.9249 & 4.4668 & 15 \\
6.4 & -14.4169 & 4.4214 & 11 & 7.14 & -16.7782 & 4.4109 & 13 \\
6.5 & -14.3826 & 4.4038 & 11 & 7.15 & -16.4594 & 4.3653 & 12 \\
6.6 & -14.1826 & 4.4335 & 9 & 8.1 & -20.2390 & 4.4852 & 17 \\
6.7 & -13.8972 & 4.3866 & 10 & 9.1 & -23.2084 & 4.4853 & 21 \\
6.8 & -13.3070 & 4.3408 & 9 & 10.1 & -26.0546 & 4.5062 & 24 \\
7.1 & -17.4335 & 4.4637 & 15 & 11.1 & -28.8222 & 4.5021 & 27 \\
7.2 & -17.3851 & 4.5238 & 15 & 12.1 & -31.6743 & 4.4937 & 29 \\
7.3 & -17.3472 & 4.5520 & 15 & 13.1 & -34.5353 & 4.5175 & 33 \\
7.4 & -17.3250 & 4.4361 & 14 & 14.1 & -37.4416 & 4.5159 & 37 \\
\hline \hline
\end{tabular}

experimental investigation. ${ }^{42}$ The properties of the ground state of the hydrogen molecule are obtained from Ref. 43. As can be seen in Table II, the agreement of most calculated properties with experiment is quite good.

\section{B. $\mathrm{Ni}_{3}-\mathrm{Ni}_{14}$}

The structures in Table III are labeled (following Stave and DePristo ${ }^{8}$ ) with two indices, N.m, where $N$ is the number of atoms and $m$ refers to a particular isomer. The isomers are sorted in ascending energy order so that $m=1$ refers to the lowest minimum found. Table III contains the binding energy for all the isomers found for $\mathrm{Ni}_{3}$ to $\mathrm{Ni}_{7}$ and for the global minima of $\mathrm{Ni}_{8}$ to $\mathrm{Ni}_{14}$. We also list the number of bonds and the average near neighbor bond length for each isomer. The number of bonds in a given cluster $N_{b}$ is calculated with a smooth cutoff function ${ }^{21}$

$$
N_{b}=\sum_{i, j>i}^{N}\left[\exp \left(\frac{r_{i, j}-r_{c}}{\delta}\right)+1\right]^{-1},
$$

where $r_{c}=5.6796788$ bohr and $\delta=0.1839$ bohr are chosen so that the coordination around an atom in the bulk fcc structure is 12.00 to four significant figures. The results reported in Table III are rounded to the nearest integer. The smooth distribution in Eq. (12) is used to calculate the average nearest neighbor bond length $\left\langle r_{e}\right\rangle$

$$
\left\langle r_{e}\right\rangle=\frac{1}{N_{b}} \sum_{i, j>i}^{N} r_{i, j}\left[\exp \left(\frac{r_{i, j}-r_{c}}{\delta}\right)+1\right]^{-1} .
$$

It can be clearly seen in Table III that $\left\langle r_{e}\right\rangle$ is a slowly increasing function of cluster size and approaches a number close to the experimental bulk Ni-Ni distance (4.72 bohr). We also see from Table III that the energy of different isomers of the same size does not depend strongly on the number of bonds, although in general, structures with higher coordination are favored. 

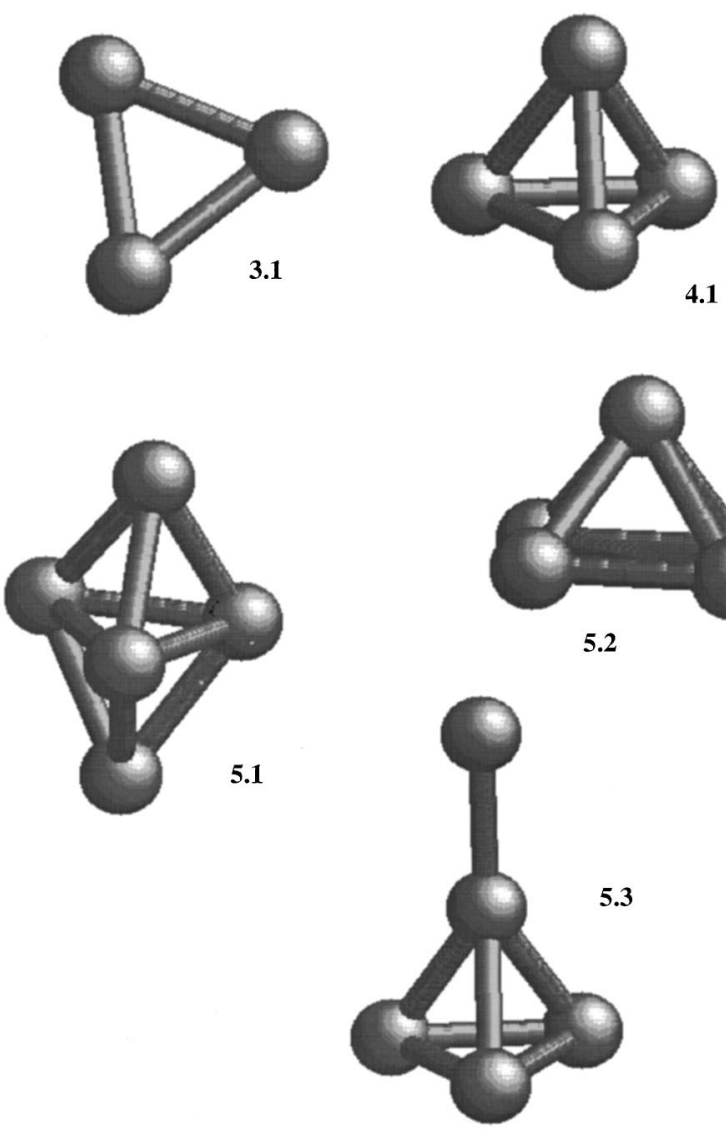

FIG. 1. All of the isomers found for $\mathrm{Ni}_{3}$ to $\mathrm{Ni}_{5} \cdot \mathrm{Ni}_{3}$ has $\mathrm{D}_{3 h}$ symmetry. $\mathrm{Ni}_{4}$ has $\mathrm{D}_{2 d}$ symmetry as is slightly distorted from the tetrahedral configuration. For the corresponding binding energies see Table III.

The structures of the isomers of $\mathrm{Ni}_{3}$ to $\mathrm{Ni}_{5}$ are reproduced in Fig. $1 . \mathrm{Ni}_{3}$ has $\mathrm{D}_{3 h}$ symmetry. $\mathrm{Ni}_{4}$ is distorted from the tetrahedral symmetry by a slight amount. Inspection of the molecular orbital energies for $\mathrm{T}_{d} \mathrm{Ni}_{4}$ reveals the reason for its distortion. The highest occupied molecular orbital in $\mathrm{T}_{d} \mathrm{Ni}_{4}$ is triply degenerate and the shell is not fully occupied. The adiabatic surface is consequently also degenerate. By the Jahn-Teller theorem ${ }^{44}$ one of these degenerate surfaces cannot have a minimum at the $\mathrm{T}_{d}$ configuration of $\mathrm{Ni}_{4}$. These distortions are observed in higher clusters as well. In these the Jahn-Teller effect greatly complicates the potential energy surface by increasing the number of lower symmetry isomers about a structure with degeneracies in the adiabatic surface. The first system that displays more than one distorted isomer is $\mathrm{Ni}_{6}$ for which we find eight different stable structures (see Fig. 2). The first two are very similar having the slight distortions of the octahedral configuration as recognized previously by $\mathrm{Yu}$ and Almöf. ${ }^{45}$ Isomers 6.5 and 6.7 contain a plane of symmetry defined by the coordinates of four atoms. For 6.5 the atom capping the trigonal bipyramid is in the same plane with the nearest equatorial atom and with the two vertices. In 6.7 the two atoms capping the tetrahedron are in the same plane with the two atoms that they coordinate. These planes divide the rest of the molecule equally. The atoms of 6.8 are not all in the plane of the page but the plane perpendicular to the page is a symmetry ele-
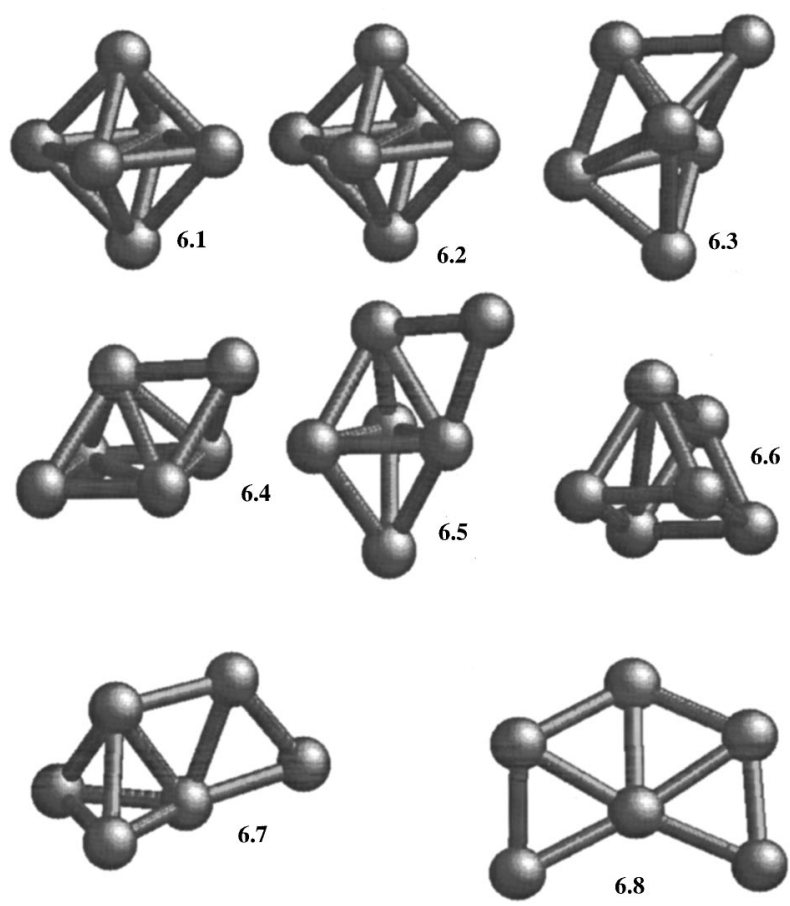

FIG. 2. All of the isomers found for $\mathrm{Ni}_{6}$. The two lowest minima are distorted octahedra. The highest isomer is slightly bent from the planar arrangement (plane of the page) but has a plane of symmetry perpendicular to the page. All isomer found for $\mathrm{Ni}_{6}$ are not chiral.

ment. Parks et al. ${ }^{29}$ find experimental evidence of rearrangements of the $\mathrm{Ni}_{6}$ substrate at relatively high $\mathrm{N}_{2}$ pressures. The lowest minimum for $\mathrm{Ni}_{6}$ proposed by the authors of this study is the octahedron in agreement with the results of both previous $\mathrm{CEM}^{8}$ and EAM models. ${ }^{5}$ The existence of the capped square pyramid has been hypothesized by Parks et al. $^{29}$ as a possible rearranged structure when one additional $\mathrm{N}_{2}$ molecule is accommodated at elevated $\mathrm{N}_{2}$ pressures.

The topology of $\mathrm{Ni}_{7}$ is even more intriguing. The lowest minimum is the capped octahedron. This is identical to the structure inferred from $\mathrm{N}_{2}$ uptake experiments. ${ }^{29}$ This close agreement with the experimental finding, however, may be fortuitous. All the isomers are very close in energy even when their geometries are quite different. We also find isomers which differ energetically but are quite close topologically. A clear example is given by isomers 7.2 and 7.3 which are shown in Fig. 3. Of the 15 minima found, 5 resemble transition states that have been reported for Lennard-Jones 7-particle systems by Tsai and Jordan. ${ }^{46}$ These are 7.2, 7.3, $7.5,7.7,7.12$, while isomer 7.6 resembles a second order saddle in $\mathrm{Ar}_{7}$.

Recently, two additional $a b$ initio calculations have been carried out on $\mathrm{Ni}_{7}$ by Nayak et al. ${ }^{47}$ The authors of this work report two structures, a capped octahedron and a pentagonal bipyramid, obtained by ab initio calculations minimized with symmetry constraints. The capped octahedron is reported by these authors to be $0.35 \mathrm{eV}$ lower in energy then the pentagonal bipyramid. In the present study, the difference be- 

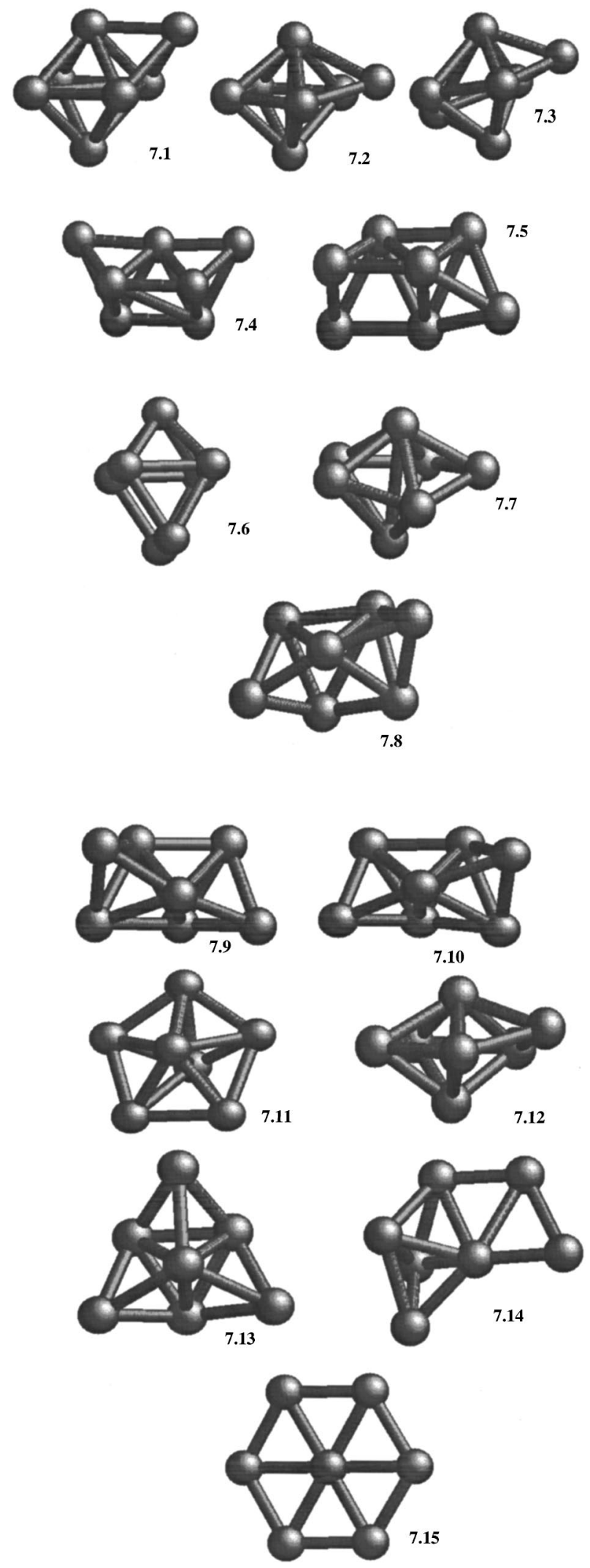

FIG. 3. All geometrical isomers of $\mathrm{Ni}_{7}$. Isomers 7.2, 7.3, 7.7, 7.11 and 7.12 are very similar geometrically but are distinct distortions of the pentagonal bipyramid. Isomers 7.9, 7.10, and 7.8 resemble the highest isomer of $\mathrm{Ar}_{7}$ and are distinct from each other. Isomer 7.3, 7.8, 7.9, 7.10, 7.13 are chiral.

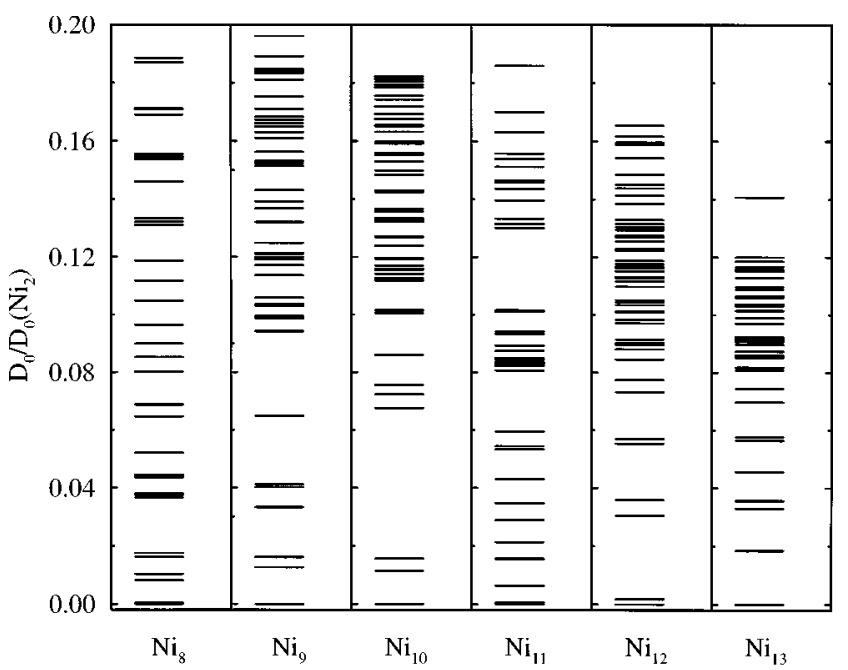

FIG. 4. Plot of the first few isomeric energies as a function of cluster size. Binding energies are rescaled so that the lowest minimum for each system is zero, and divided by the binding energy of the dimer $D^{0}\left(\mathrm{Ni}_{2}\right)$.

tween the most symmetric pentagonal bipyramid (7.11 in Fig. 3) and the capped octahedron is found to be $0.24 \mathrm{eV}$ in good agreement with these recent findings.

The structures 7.8, 7.9, 7.10, can be thought of as missed pentagonal bipyramids where one of the equatorial atoms is on a face of the structure or as capped trigonal bipyramids. The structure of these minima resemble the highest isomer of a 7-particle Lennard-Jones cluster. Isomers 7.3, 7.8, 7.9, $7.10,7.13$ are chiral. The respective enantiomers have been found for each of these.

Beginning with $\mathrm{Ni}_{8}$, the number of minima becomes quite large. Rather than tabulate the energies for all the lowest structures we report a plot of the local minima in the potential surface found as a function of cluster size $N$. This graph is displayed in Fig. 4. In Fig. 4 we have not included more than 1 line for structures that have optical isomers of equivalent energy. To emphasize the small differences in energy between isomers we shift the energies so that the lowest minimum found for each system is zero and we divide the resulting binding energy for each structure by the binding energy of the dimer $D^{0}\left(\mathrm{Ni}_{2}\right)$. All the minima considered are within $0.2 D^{0}\left(\mathrm{Ni}_{2}\right)$ and are distributed quite irregularly. This picture demonstrates one of the differences that exist between the minima of transition metal clusters and LennardJones clusters. In the latter system the gap between the lowest isomer and the next one is typically on the order of one bond in the size range considered in the present study. (The 17 atom Lennard-Jones cluster is an exception, where the three distinct lowest energy isomers have been found that have the same coordination and are very close in energy. ${ }^{48}$ ) Figure 4 also demonstrates that the density of isomeric states increases with size as is expected. The isomers in this size range reveal the presence of the same features found in $\mathrm{Ni}_{6}$ and $\mathrm{Ni}_{7}$, namely, a number of minima are very similar geometrically and differ from one another from distortions which lower the symmetry of one or more unstable configurations. 


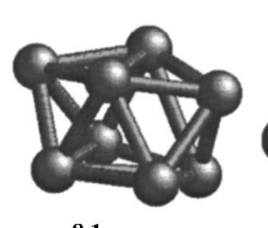

8.1
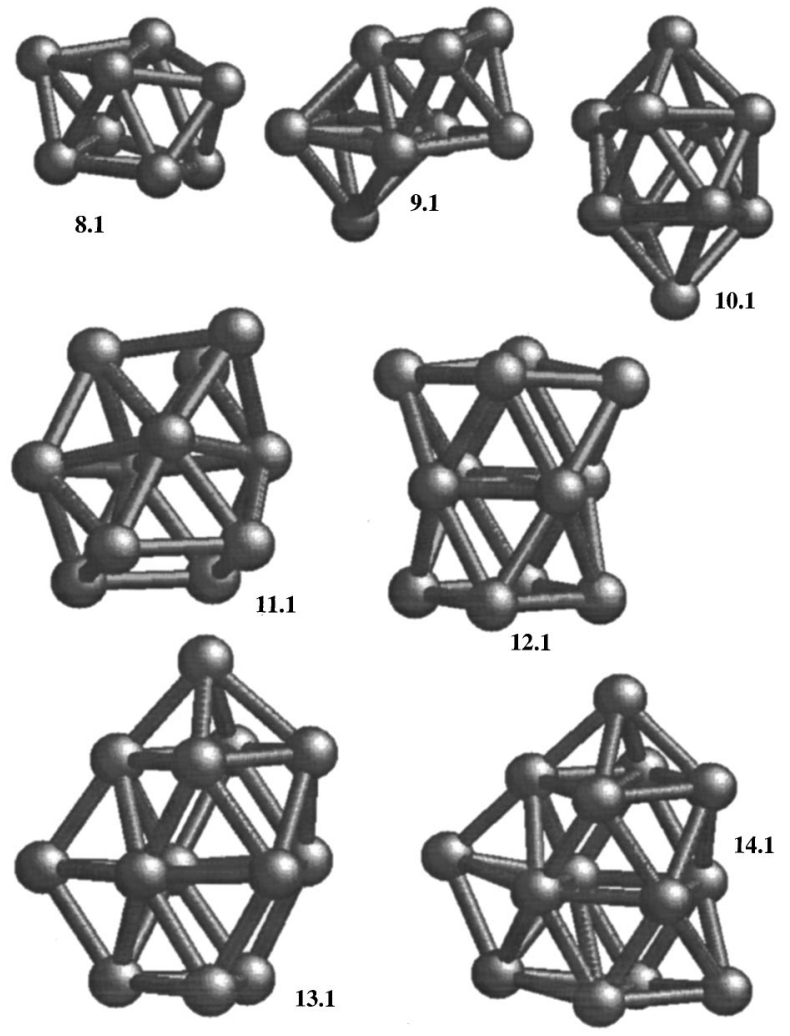

FIG. 5. The lowest geometrical isomer found for $\mathrm{Ni}_{8}$ to $\mathrm{Ni}_{14} \cdot \mathrm{Ni}_{10}$, is a capped square antiprism, $\mathrm{Ni}_{11}$ is a capped trigonal prism, $\mathrm{Ni}_{12}, \mathrm{Ni}_{13}, \mathrm{Ni}_{14}$ are built from a square antiprism interpenetrating a trigonal prisms. One of the basis of the square antiprism shares the same 4 atoms of the 4-atom face of the trigonal prisms. $\mathrm{Ni}_{9}$ is a fragment of a hep crystal.

In Fig. 5 we display the lowest minima of $\mathrm{Ni}_{8}$ to $\mathrm{Ni}_{14}$. The lowest minimum for $\mathrm{Ni}_{8}$ is a 4,4 capped trigonal prism. ${ }^{49}$ For $\mathrm{Ni}_{8}$ we also find the bisdisphenoid $\mathrm{D}_{2 d}$ (which is higher in energy only by $0.01 \mathrm{eV}$ ), the square antiprism and the capped pentagonal bipyramid. It is noted that both the bisdisphenoid and the square antiprism are mentioned as plausible candidates by Parks et al. ${ }^{29}$ who, however, indicate that the capped pentagonal bipyramid may be the global minimum for $\mathrm{Ni}_{8}$. Stave and DePristo ${ }^{8}$ report the bisdisphenoid as the lowest minimum of $\mathrm{Ni}_{8}$. The lowest minimum of $\mathrm{Ni}_{9}$ is a twin trigonal antiprism reproduced in Fig. 5. This particular structure is a fragment of a hcp crystal and can also be thought of as two fused octahedra. Close to the global minima we also find the 4,4,4 capped trigonal prism and the 4 capped square antiprism. We also find the capped pentagonal bipyramid, $0.09 \mathrm{eV}$ above the lowest minimum and at lower energies capped octahedra and fcc or hcp fragments. The lowest minimum for $\mathrm{Ni}_{10}$ is a 4,4 capped square antiprism. $\mathrm{Ni}_{11}$ global minimum is a distorted capped trigonal prism, $\mathrm{Ni}_{12}$ is like $\mathrm{Ni}_{10}$ a capped square antiprism.

$\mathrm{Ni}_{13}$ is a capped square antiprism interpenetrating a capped trigonal prism. The tetrahedron capped by an equilateral triangle (hcp fragment) minimizes $0.1 \mathrm{eV}$ above the lowest minimum. The icosahedron minimizes $0.26 \mathrm{eV}$ above and the cuboctahedron $0.52 \mathrm{eV}$ above the lowest minimum. From Fig. 5 it is evident that the growth pattern is nontrivial. We

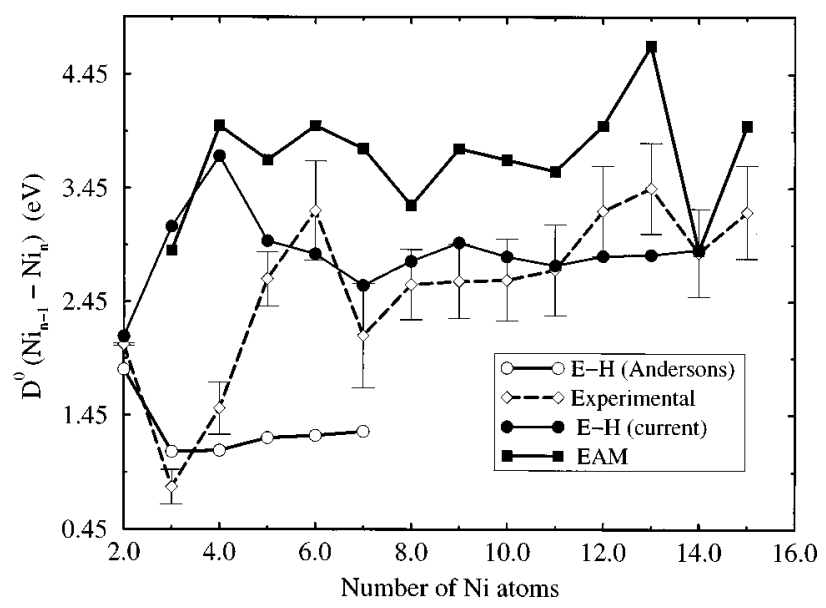

FIG. 6. Graph of $D^{0}\left(\mathrm{Ni}_{n-1}\right)-D^{0}\left(\mathrm{Ni}_{n}\right)$ as a function of $n$. The dashed line with opened diamonds is the experimental data of Ref. 28, the dark circles with a solid line are our results, the open circles with a solid line are our results using Anderson's (Ref. 12) parameters. The dark squares are previous EAM results (Ref. 5).

find that icosahedral growth is not favored in this size range. The global minima we find better resemble the growth of closo $\mathrm{B}_{n} \mathrm{H}_{n}^{2-}$ inorganic anions, ${ }^{50}$ but there are obvious exceptions. While icosahedral structures are found among the isomers, the present model does not predict that the global minimum is obtained by maximizing the number of $\mathrm{Ni}-\mathrm{Ni}$ near-neighbor interactions as is the case for Lennard-Jones clusters. ${ }^{51}$ Rather, a preference to coordinate more uniformly every atom in the cluster is observed. This observation is the same that Stave and DePristo ${ }^{8}$ make in their study.

In Fig. 6 we plot the energy difference between lowest minima of clusters of adjacent size. Since the reference energy of the single $\mathrm{Ni}$ atom is zero, the quantity plotted is the energy differences associated with the gas phase reaction

TABLE IV. Potential energy of the lowest minima found for nickel clusters with one hydrogen.

\begin{tabular}{ccc}
\hline \hline Structure & Isomer & $\mathrm{V}(\mathrm{eV})$ \\
\hline $\mathrm{Ni}_{2} \mathrm{H}$ & 1 & -5.3733 \\
$\mathrm{Ni}_{2} \mathrm{H}$ & 2 & -5.3732 \\
$\mathrm{Ni}_{2} \mathrm{H}$ & 3 & -5.3324 \\
$\mathrm{Ni}_{2} \mathrm{H}$ & 4 & -5.0253 \\
$\mathrm{Ni}_{3} \mathrm{H}$ & 1 & -8.6277 \\
$\mathrm{Ni}_{4} \mathrm{H}$ & 1 & -12.1315 \\
$\mathrm{Ni}_{4} \mathrm{H}$ & 2 & -11.9105 \\
$\mathrm{Ni}_{5} \mathrm{H}$ & 1 & -14.9679 \\
$\mathrm{Ni}_{5} \mathrm{H}$ & 2 & -14.8478 \\
$\mathrm{Ni}_{5} \mathrm{H}$ & 3 & -14.7867 \\
$\mathrm{Ni}_{5} \mathrm{H}$ & 4 & -14.7686 \\
$\mathrm{Ni}_{5} \mathrm{H}$ & 5 & -14.7522 \\
$\mathrm{Ni}_{5} \mathrm{H}$ & 6 & -14.9491 \\
$\mathrm{Ni}_{6} \mathrm{H}$ & 1 & -17.8187 \\
$\mathrm{Ni}_{7} \mathrm{H}$ & 1 & -20.5256 \\
$\mathrm{Ni}_{8} \mathrm{H}$ & 1 & -23.3720 \\
$\mathrm{Ni}_{9} \mathrm{H}$ & 1 & -26.2703 \\
$\mathrm{Ni}_{10} \mathrm{H}$ & 1 & -29.0961 \\
$\mathrm{Ni}_{11} \mathrm{H}$ & 1 & -31.7802 \\
\hline \hline
\end{tabular}


TABLE V. Geometry of $\mathrm{Ni}_{2} \mathrm{H}$ Isomer 1 .

\begin{tabular}{lcc}
\hline \hline \multicolumn{3}{c}{ Bond lengths $(\AA)$} \\
\hline $\mathrm{Ni}(1)-\mathrm{Ni}(2)$ & & 2.26487 \\
$\mathrm{Ni}(1)-\mathrm{H}$ & & 1.74371 \\
$\mathrm{Ni}(2)-\mathrm{H}$ & 1.50610 \\
& Bond angles (degrees) & \\
\hline $\mathrm{Ni}(1)-\mathrm{Ni}(2)-\mathrm{H}$ & & 50.3 \\
$\mathrm{Ni}(1)-\mathrm{H}-\mathrm{Ni}(2)$ & & 88.0 \\
$\mathrm{Ni}(2)-\mathrm{Ni}(1)-\mathrm{H}$ & & 41.6 \\
\hline \hline
\end{tabular}

$$
\mathrm{Ni}_{n} \rightarrow \mathrm{Ni}_{n-1}+\mathrm{Ni} \quad \Delta E_{n}=D^{0}\left(\mathrm{Ni}_{n-1}\right)-D^{0}\left(\mathrm{Ni}_{n}\right) .
$$

Included in Fig. 6 are experimental results derived from the scattering of nickel clusters ions with Xe atoms. ${ }^{28}$ These experimental results are depicted as the dashed line with open diamonds in Fig. 6. The table in this Ref. 28 is recalculated using only the first atomization energy before comparing this to our results. We compare our calculation (filled circles, dark solid line) to the experimental results. It is noted that with the exception of $\mathrm{Ni}_{3}$ and $\mathrm{Ni}_{4}$ our estimation of $\Delta E_{n}$ is nearly within the experimental error bars. As mentioned previously, this is not the case if we use the orbital parameters of Ref. 12 (open circles, dark solid line). The lack of structure in Fig. 6 when Anderson's parameters are used has led us to the re-parameterization discussed in this work.

\section{C. $\mathrm{Ni}_{2} \mathrm{H}$ to $\mathrm{Ni}_{11} \mathrm{H}$}

In Table IV we list the binding energy of all discovered isomers of singly hydrogenated nickel cluster up to $\mathrm{Ni}_{5} \mathrm{H}$ (for which the search appears to be comprehensive) and the lowest minimum found for all the remaining clusters examined in this work. As can be seen in Table IV, $\mathrm{Ni}_{2} \mathrm{H}$ has four minima. The first three are rings, the fourth is a nonlinear chain. The differences between the lowest three isomers are small. The lowest minimum of $\mathrm{Ni}_{2} \mathrm{H}$ is distorted from $\mathrm{C}_{2 v}$ symmetry. The two Ni-H bonds are 3.2948 and 2.8460 bohr, respectively and the $\mathrm{Ni}-\mathrm{Ni}$ bond is $2 \%$ longer than in $\mathrm{Ni}_{2}$ alone. The next two structures are also distorted from $\mathrm{C}_{2 v}$ symmetry, but by a smaller amount. Because the isomers are so close geometrically, we characterize fully their geometries in Table V-VIII. Each minimum is verified by Brownian dynamics and Monte-Carlo simulated annealing to one part in $10^{12}$. All $3 N-6$ eigenvalues of the Hessian matrix are positive for each structure, indicating that these are not

TABLE VI. Geometry of $\mathrm{Ni}_{2} \mathrm{H}$ Isomer 2 .

\begin{tabular}{lcc}
\hline \multicolumn{3}{c}{ Bond lengths $(\AA)$} \\
\hline $\mathrm{Ni}(1)-\mathrm{Ni}(2)$ & 2.27734 \\
$\mathrm{Ni}(1)-\mathrm{H}$ & 1.57045 \\
$\mathrm{Ni}(2)-\mathrm{H}$ & 1.57041 \\
& Bond angles (degrees) \\
\hline $\mathrm{Ni}(1)-\mathrm{Ni}(2)-\mathrm{H}$ & \\
$\mathrm{Ni}(1)-\mathrm{H}-\mathrm{Ni}(2)$ & 43.5 \\
$\mathrm{Ni}(2)-\mathrm{Ni}(1)-\mathrm{H}$ & 92.9 \\
\hline \hline
\end{tabular}

TABLE VII. Geometry of $\mathrm{Ni}_{2} \mathrm{H}$ Isomer 3.

\begin{tabular}{lcc}
\hline \hline \multicolumn{3}{c}{ Bond length $(\AA)$} \\
\hline $\mathrm{Ni}(1)-\mathrm{Ni}(2)$ & & 2.19581 \\
$\mathrm{Ni}(1)-\mathrm{H}$ & & 1.88531 \\
$\mathrm{Ni}(2)-\mathrm{H}$ & 1.88539 \\
& Bond angles (degrees) \\
\hline $\mathrm{Ni}(1)-\mathrm{Ni}(2)-\mathrm{H}$ & & \\
$\mathrm{Ni}(1)-\mathrm{H}-\mathrm{Ni}(2)$ & & 71.4 \\
$\mathrm{Ni}(2)-\mathrm{Ni}(1)-\mathrm{H}$ & 54.4 \\
\hline \hline
\end{tabular}

saddles. To interpret the distortions we inspect the energy levels of a perfect $\mathrm{C}_{2 v} \mathrm{Ni}_{2} \mathrm{H}$. This structure is constructed with the $\mathrm{Ni}-\mathrm{Ni}$ and $\mathrm{Ni}-\mathrm{H}$ bond lengths equal to the respective dimers. The highest occupied shell is a doubly degenerate electronic orbital and is partially filled. The $\mathrm{C}_{2 v}$ point group has no degenerate irreducible representations. In this case, however, the degeneracy of the molecular orbitals comes from two pairs (one filled and one partially filled) of molecular orbitals (MO) that consist entirely of $p$ and $d$ functions of the $\mathrm{Ni}$ atoms. The $1 s$ orbital of hydrogen is centered exactly in a nodal plane and yields no contribution to the bonding in these degenerate pairs of MOs. As the hydrogen atom is moved away from the nodal plane one of the orbitals of the degenerate pairs of MOs acquires significant contribution from the $1 s$ orbital resulting in a lowering of its energy. This observed degeneracy in the $\mathrm{C}_{2 v}$ configuration and its resulting distortion is the result of restricting the atomic basis set on the hydrogen atom. This artifact may explain the unusually large number of isomers that we find for this cluster. We are unable to predict the number of isomers expected if a more complete basis set were used breaking the degeneracy. In contrast to $\mathrm{Ni}_{2} \mathrm{H}$ we find only one isomer of $\mathrm{Ni}_{3} \mathrm{H}$; a distorted trigonal pyramid. Planar $\mathrm{Ni}_{3} \mathrm{H}$ is not stable. The distortion of $\mathrm{Ni}_{3} \mathrm{H}$ is again interpreted by inspection of the energy levels of $\mathrm{C}_{3 v} \mathrm{Ni}_{3} \mathrm{H}$. The highest occupied orbital shell is doubly degenerate and only partially filled.

$\mathrm{Ni}_{4} \mathrm{H}$ only has two isomers. The lowest has $\mathrm{C}_{2 v}$ symmetry and is reproduced in Fig. 7. Inspection of the electronic energy levels indicates that this structure should not be distorted as no degeneracies in the adiabatic surface exist. In contrast to $\mathrm{Ni}_{3} \mathrm{H}$ we do not find a face capping configuration, even when distorted. The potential energy surface of $\mathrm{Ni}_{4} \mathrm{H}$ at the $\mathrm{C}_{3 v}$ configuration (when the $\mathrm{H}$ atoms caps any one of the tetrahedral faces most symmetrically) is doubly degenerate.

TABLE VIII. Geometry of $\mathrm{Ni}_{2} \mathrm{H}$ Isomer 4 .

\begin{tabular}{lc}
\hline \multicolumn{3}{c}{ Bond length $(\AA)$} \\
\hline $\mathrm{Ni}(1)-\mathrm{Ni}(2)$ & 2.21532 \\
$\mathrm{Ni}(1)-\mathrm{H}$ & 3.18447 \\
$\mathrm{Ni}(2)-\mathrm{H}$ & 1.51123 \\
& \\
\hline $\mathrm{Ni}(1)-\mathrm{Ni}(2)-\mathrm{H}$ & Bond angles (degrees) \\
$\mathrm{Ni}(1)-\mathrm{H}-\mathrm{Ni}(2)$ & 116.1 \\
$\mathrm{Ni}(2)-\mathrm{Ni}(1)-\mathrm{H}$ & 38.6 \\
\hline \hline
\end{tabular}




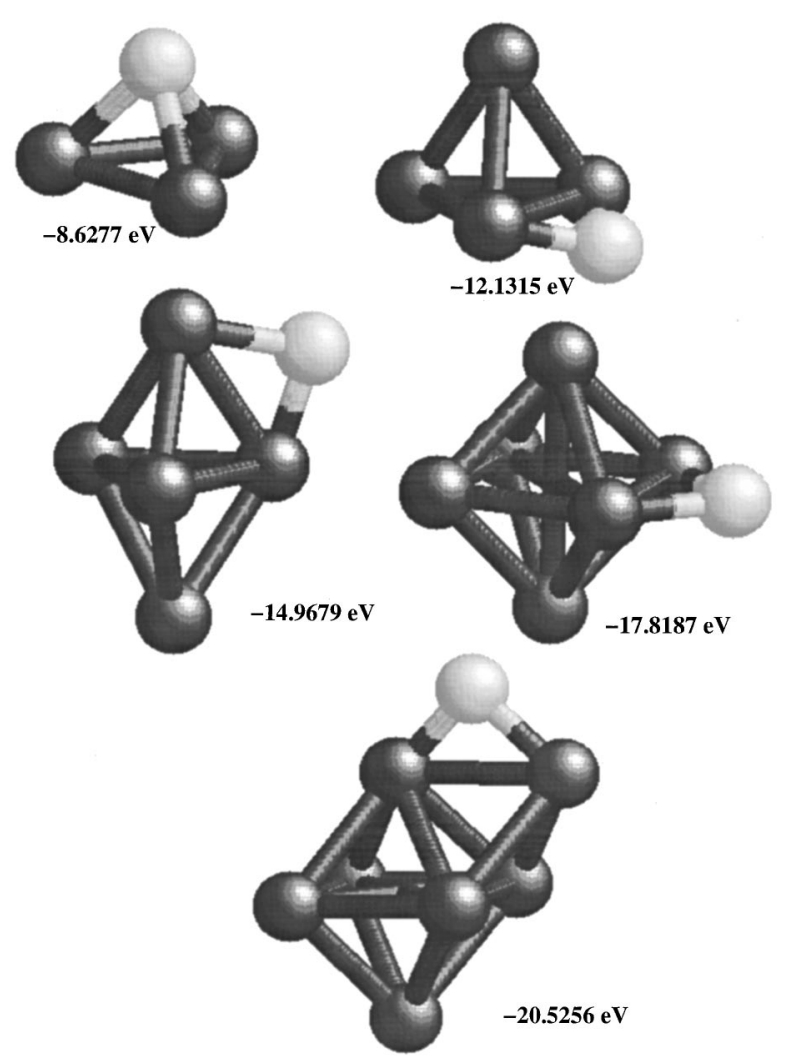

FIG. 7. Lowest minimum found for $\mathrm{Ni}_{3} \mathrm{H}$ to $\mathrm{Ni}_{7} \mathrm{H}$. The hydrogen atom is depicted with a lighter shading.

The second isomer of $\mathrm{Ni}_{4} \mathrm{H}$ is a distorted tetrahedral $\mathrm{Ni}_{4}$ with a hydrogen sitting on top. This structure resembles the third isomer of $\mathrm{Ni}_{5}$ in Fig. 1. Unlike recent findings ${ }^{5}$ with the embedded atom model, we do not find any $\mathrm{H}$ atoms in the tetrahedral site. The tetrahedral configuration of $\mathrm{Ni}_{4} \mathrm{H}$ should distort as consequence of an orbital degeneracy. The $\mathrm{Ni}-\mathrm{H}$ distances in the $\mathrm{D}_{2 d}$ structure with $\mathrm{H}$ in the center of mass (which derives from the distortion of $\mathrm{T}_{d} \mathrm{Ni}_{4} \mathrm{H}$ ), are all slightly larger than the $\mathrm{Ni}-\mathrm{H}$ equilibrium distance of the dimer. Nevertheless, the repulsive part of the potential has a maximum at this configuration and the electronic part of the energy is not sufficiently deep to stabilize the configuration.

The lowest minimum of $\mathrm{Ni}_{5} \mathrm{H}$ is reproduced in Fig. 7. The structure has a plane of symmetry containing the $\mathrm{H}$ atom, the atoms at the two vertices of the trigonal bipyramid, and the equatorial atom bonded to hydrogen. For $\mathrm{Ni}_{5} \mathrm{H}$ we find all the isomers for the corresponding bare cluster with the same energetic rank. In each, the bridging site $(\mathrm{H}$ atom bonding symmetrically two Ni atoms) is preferred energetically to the threefold site and this is in turn preferred to the single Ni-H interaction. The only exception is in the third isomer of $\mathrm{Ni}_{5}$, where only a capped face is found.

As with the bare clusters, beginning with $\mathrm{Ni}_{6} \mathrm{H}$ we do not tabulate the potential energy for every isomer found. Rather, we report the energy of the lowest minimum for each in Table IV and a graph of the energy of the isomers as function of number of $\mathrm{Ni}$ atoms in Fig. 9. The addition of a hydrogen atom effects the isomer ordering of the nickel sub-

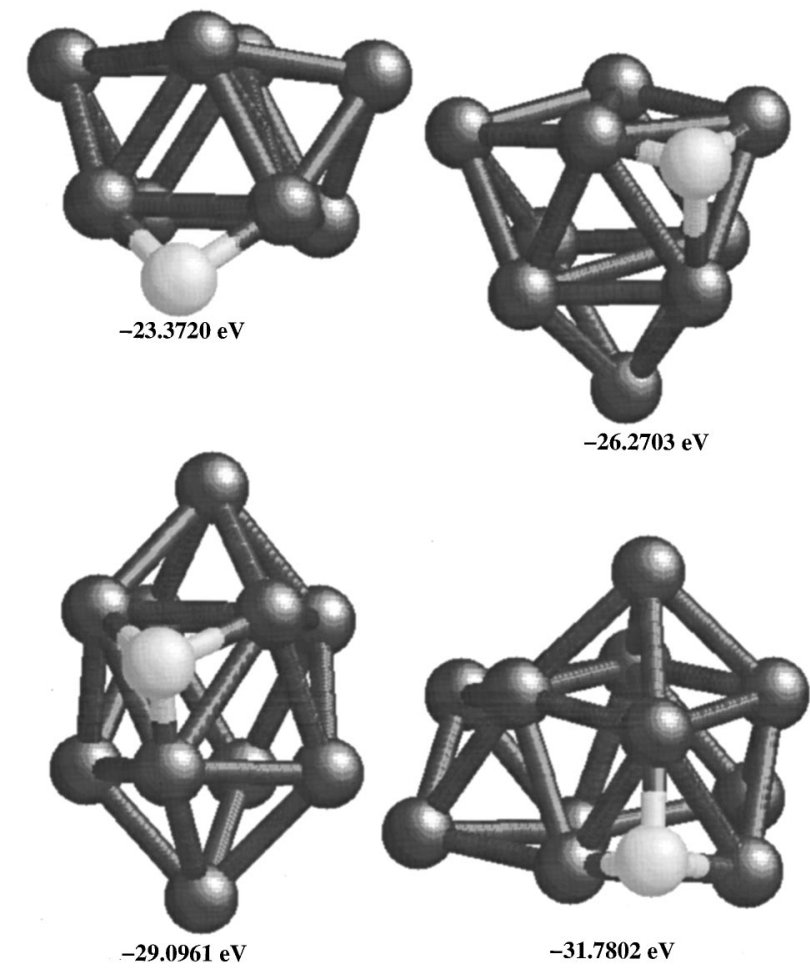

FIG. 8. Lowest minimum found for $\mathrm{Ni}_{8} \mathrm{H}$ to $\mathrm{Ni}_{11} \mathrm{H}$.

strate in this size range. This is most evident in $\mathrm{Ni}_{9} \mathrm{H}$ in Fig. 8. The nickel substrate of $\mathrm{Ni}_{9} \mathrm{H}$ ranks higher energetically in the bare system. $\mathrm{Ni}_{8} \mathrm{H}$ and $\mathrm{Ni}_{9} \mathrm{H}$ are hydrogenated capped trigonal prisms. $\mathrm{Ni}_{11} \mathrm{H}$ is a capped square antiprism. In Fig. 10 we plot the energy of the reaction

$$
\mathrm{Ni}_{n}+\mathrm{H} \rightarrow \mathrm{Ni}_{n} \mathrm{H},
$$

at zero Kelvin as function of $n$. The first drop from $n=1$ to $n=2$ can be easily understood in terms of the difference that exists between the energies of top binding (which is the only

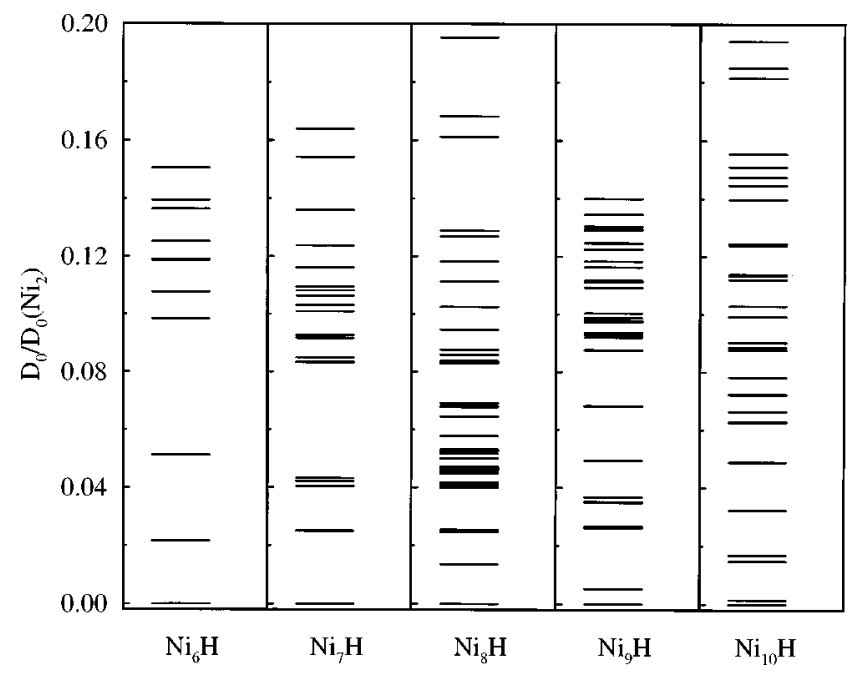

FIG. 9. Plot of the first few isomeric energy levels of $\mathrm{Ni}_{n} \mathrm{H}$ as a function of $n$. Isomer energies are rescaled so that the global minimum for each cluster size is zero, and divided by the size of the binding energy of $\mathrm{Ni}_{2}$. 


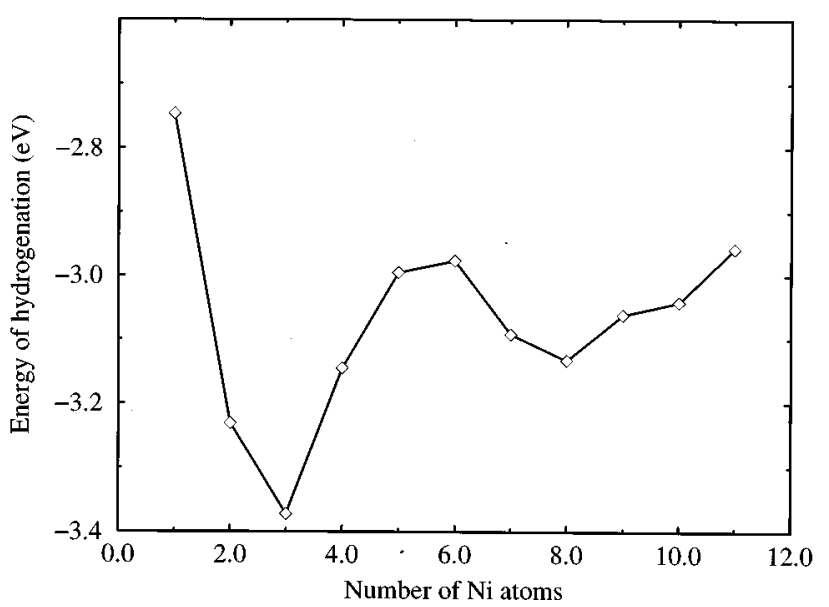

FIG. 10. Graph of the energy of hydrogenation of nickel clusters as function of cluster size.

possibility in $\mathrm{NiH}$ ) and a bridge binding site which is favored over the top configuration (bent isomer) in $\mathrm{Ni}_{2} \mathrm{H}$. The graph in Fig. 10 and the structures in Fig. 8 suggest that the difference between the two- and the threefold site decreases with $n$. This difference is much smaller than that which exists between the top site and the twofold site. The trend we observe in Fig. 7 and 8 is that larger clusters favor the threefold site to the twofold one. Ab initio calculations ${ }^{52}$ for the (111) $\mathrm{Ni}$ surface confirm that the threefold site is favored in the bulk. A slow downward trend is evident in the binding energy in Fig. 10 from $n \geqslant 3$. For $n=11$ there is already favorable agreement between the calculated energy for reaction (15) and the corresponding experimental ${ }^{53}$ value for fcc $\mathrm{Ni}$ (111), (100) and (110) of $2.7 \mathrm{eV}$.

\section{D. $\mathrm{NiH}_{2}$ to $\mathrm{Ni}_{10} \mathrm{H}_{2}$}

In all the isomers that we find for the di-hydrogenated nickel clusters, the $\mathrm{H}_{2}$ molecule is dissociated on the nickel substrate. Here too the hydrogens are predominantly on the surface. The preferred arrangement of the two $\mathrm{H}$ atoms on the surface is in two neighboring (twofold - twofold or twofold - threefold) sites, so that one $\mathrm{Ni}$ atom coordinates to both hydrogens. The only exception is found in $\mathrm{Ni}_{4} \mathrm{H}_{2}$ whose four isomers are displayed in Fig. 13. $\mathrm{NiH}_{2}$ has one stable configuration with $\mathrm{D}_{\infty h}$ symmetry. In Fig. 11 we display the structure and the energies in $\mathrm{eV}$ of the 7 isomers of $\mathrm{Ni}_{2} \mathrm{H}_{2}$. The first four structures in this figure are planar. The high number of minima for this system is a consequence of the added complexity due to the distortions from $\mathrm{C}_{2 v}$ symmetry just as is observed in $\mathrm{Ni}_{2} \mathrm{H}$. The second isomer of $\mathrm{Ni}_{3} \mathrm{H}_{2}$ in Fig. 12 is optically active. This brings the count of stable minima for $\mathrm{Ni}_{3} \mathrm{H}_{2}$ to six. This number, however, is smaller if compared with $\mathrm{Ni}_{2} \mathrm{H}_{2}$ but also parallels what is found for $\mathrm{Ni}_{2} \mathrm{H}, \mathrm{Ni}_{3} \mathrm{H}$ and $\mathrm{Ni}_{4} \mathrm{H}$. The number of isomers becomes large for $\mathrm{Ni}_{5} \mathrm{H}_{2}$. The binding energies of the lowest minima for all the di-hydrogenated system we consider are given in Table IX and the structures are reproduced in Fig. 14. The global minima for $\mathrm{Ni}_{7} \mathrm{H}_{2}, \mathrm{Ni}_{9} \mathrm{H}_{2}$ and $\mathrm{Ni}_{10} \mathrm{H}_{2}$ are hydroge-

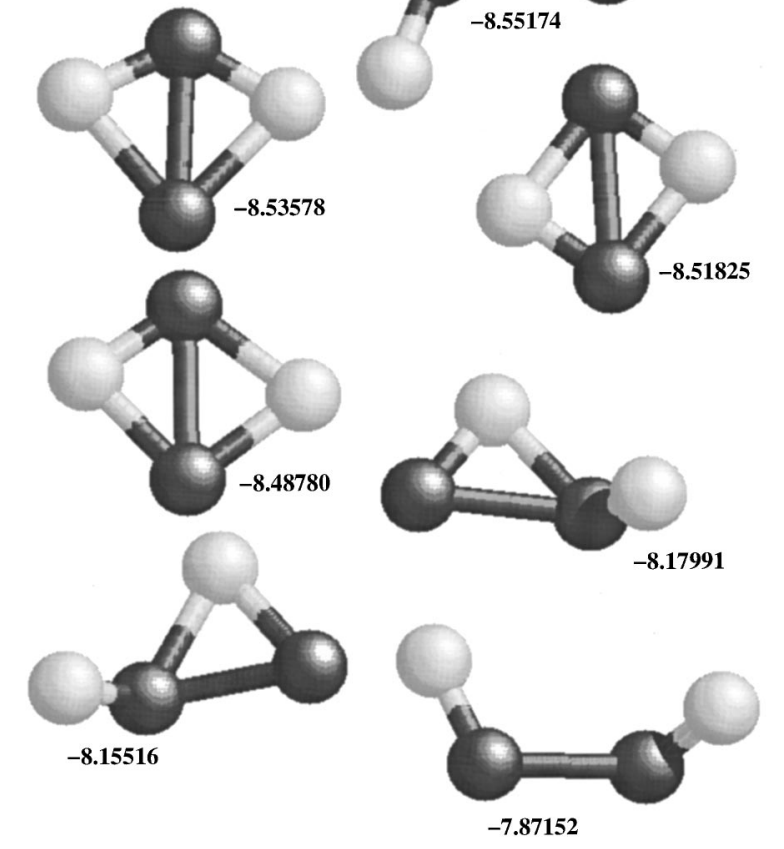

FIG. 11. All the geometric isomers found for $\mathrm{Ni}_{2} \mathrm{H}_{2}$. Energies are in eV.

nated isomers of nickel which rank higher in energy in the bare clusters. The energy of the lowest isomers found for the dehydrogenated system with 5 to 10 nickel atoms are reproduced graphically in Fig. 15.
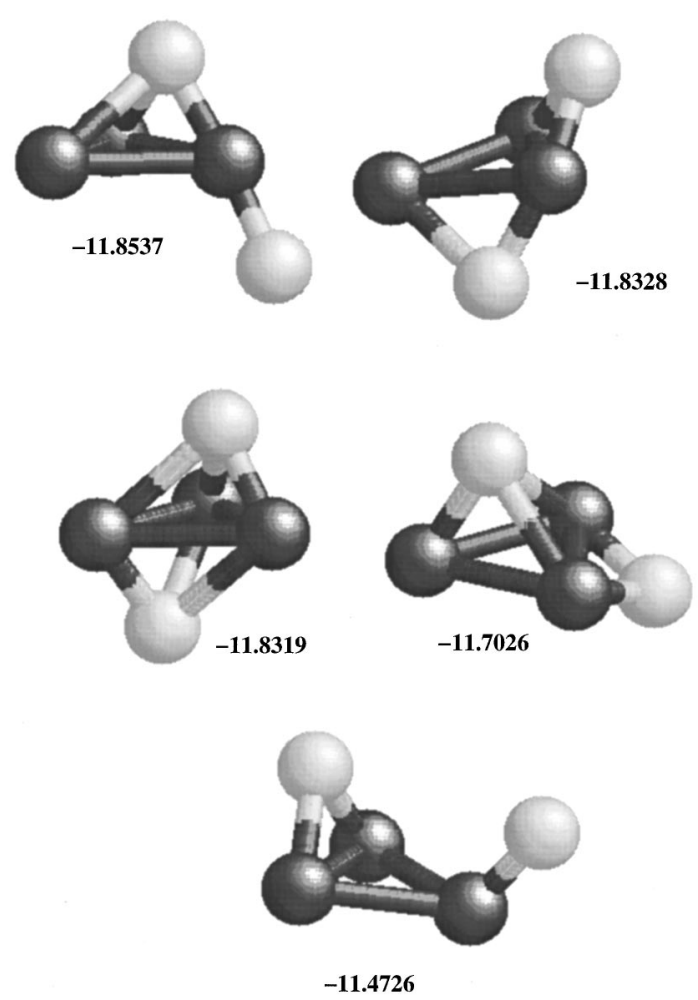

FIG. 12. All the geometric isomers found for $\mathrm{Ni}_{3} \mathrm{H}_{2}$. Energies are in eV. 

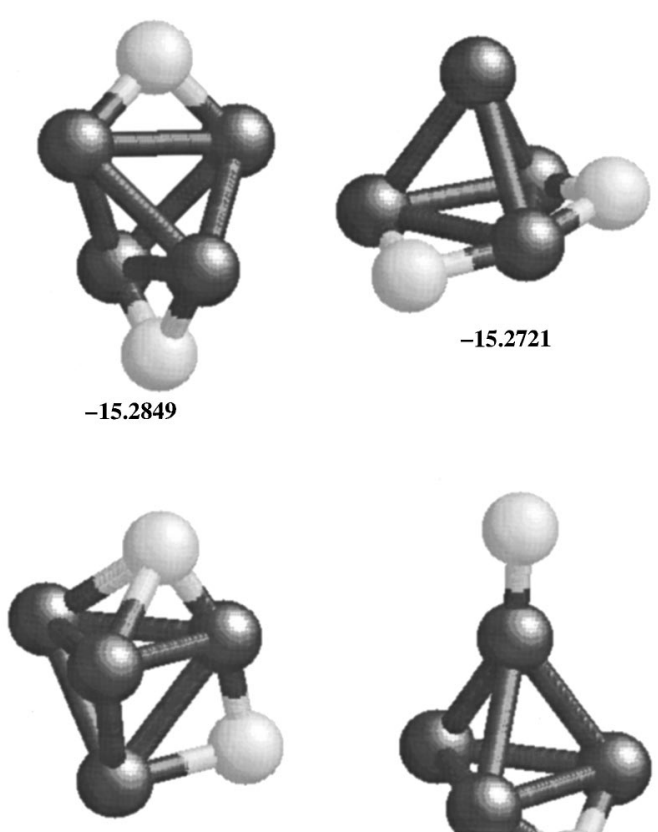

$-15.2642$

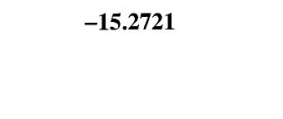

FIG. 13. All the geometric isomers found for $\mathrm{Ni}_{4} \mathrm{H}_{2}$. Energies are in $\mathrm{eV}$.

\section{DISCUSSION}

In this paper we have developed a semi-empirical potential energy model based on the extended-Hückel theory for transition metal clusters. The potential can be used in dynamic simulations since it is constructed without any bond counting terms. To eliminate these terms, optimization of the parameters is necessary. This optimization consists of two steps. The first adjustment of the parameters is a least-square fitting of binding energies between calculated and $a b$ initio results. The second step involves fitting with empirical data. Adjustment of the parameters is not automated in this step. The optimization in this manner is computationally more involved because at each step of the procedure the lowest minima of each system are required. Final geometries are sensitive to the values of the parameters.

TABLE IX. Potential energy of the lowest minima of nickel clusters with two hydrogen atoms.

\begin{tabular}{lc}
\hline \hline Cluster & $\mathrm{V}(\mathrm{eV})$ \\
\hline $\mathrm{NiH}_{2}$ & -5.5471 \\
$\mathrm{Ni}_{2} \mathrm{H}_{2}$ & -8.5517 \\
$\mathrm{Ni}_{3} \mathrm{H}_{2}$ & -11.8537 \\
$\mathrm{Ni}_{4} \mathrm{H}_{2}$ & -15.2849 \\
$\mathrm{Ni}_{5} \mathrm{H}_{2}$ & -18.2260 \\
$\mathrm{Ni}_{6} \mathrm{H}_{2}$ & -21.1350 \\
$\mathrm{Ni}_{7} \mathrm{H}_{2}$ & -23.7246 \\
$\mathrm{Ni}_{8} \mathrm{H}_{2}$ & -26.5779 \\
$\mathrm{Ni}_{9} \mathrm{H}_{2}$ & -29.4494 \\
$\mathrm{Ni}_{10} \mathrm{H}_{2}$ & -32.2195 \\
\hline \hline
\end{tabular}
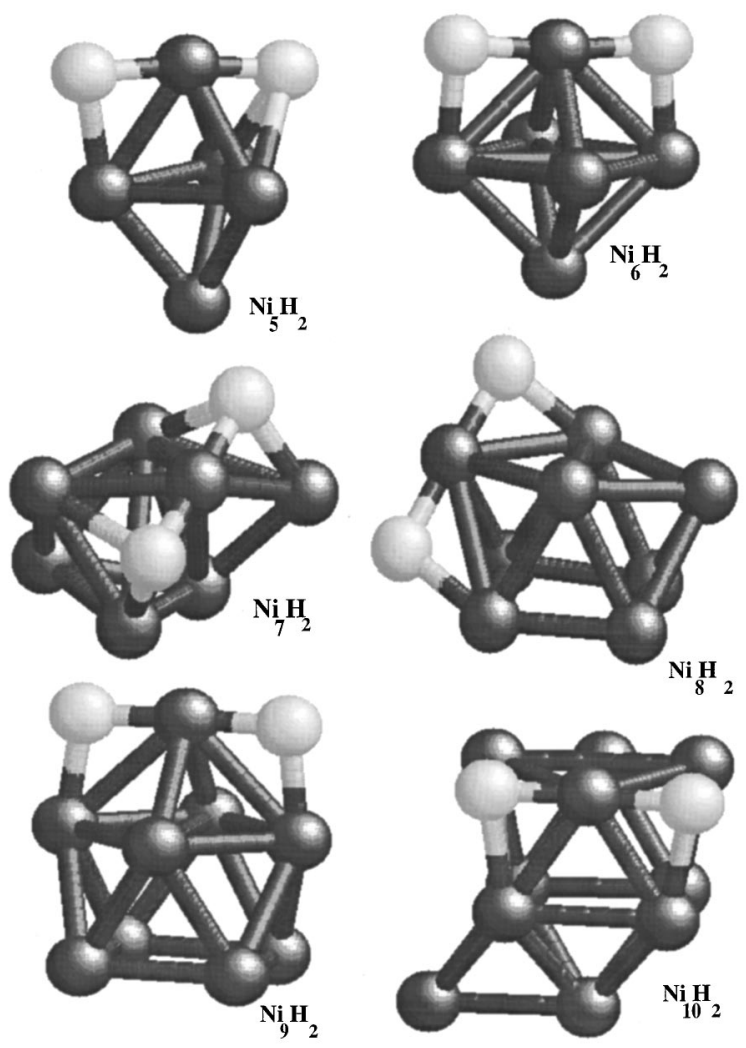

FIG. 14. Lowest minima found for $\mathrm{Ni}_{5} \mathrm{H}_{2}$ to $\mathrm{Ni}_{10} \mathrm{H}_{2} . \mathrm{Ni}_{7} \mathrm{H}_{2}$ is a distorted pentagonal bipyramid (isomer 7.2 in the bare system). $\mathrm{Ni}_{8} \mathrm{H}_{2}$ is a capped trigonal prism.

An extensive search for the minima for several different sizes of bare, mono and dehydrogenated nickel clusters has been then carried out. We find that for transition metal clusters the binding energy difference between isomers is much smaller than the dissociation energy of $\mathrm{Ni}_{2}$, and the gap decreases further in mono and dehydrogenated systems. Further, this difference is smaller than that found with EAM

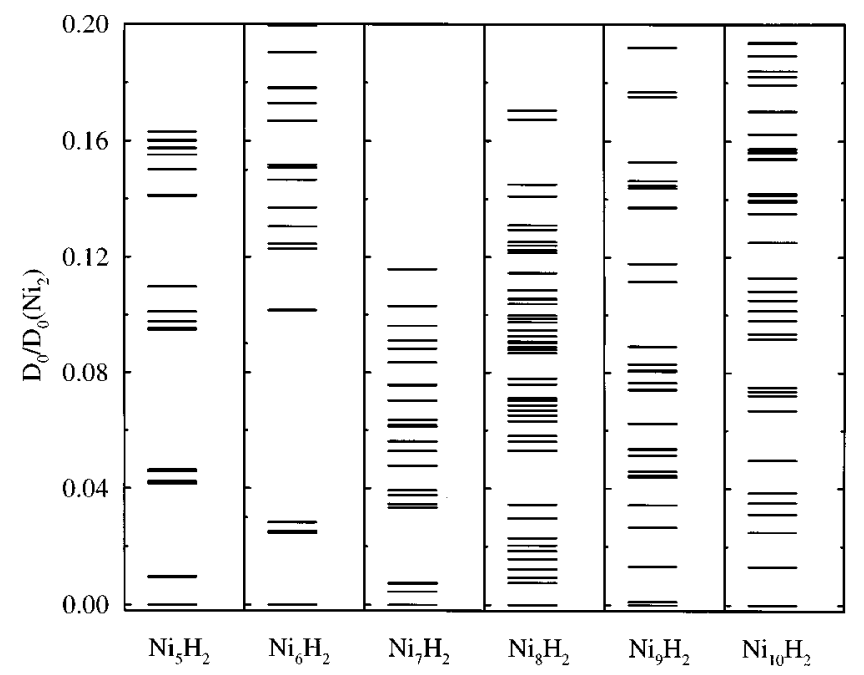

FIG. 15. Plot of the first few isomeric energy levels of $\mathrm{Ni}_{n} \mathrm{H}_{2}$ as a function of $n$. Isomer energies are rescaled so that the global minimum for each cluster size is zero, and divided by the size of the binding energy of $\mathrm{Ni}_{2}$. 
potentials. The number of minima found is large even in relatively small clusters in contrast to what is found for other models. Several structures that are stable minima with the present model resemble transition states or higher order saddles in Lennard-Jones systems. We speculate that the inclusion of the angular dependence about each nuclear center in the potential model is responsible for this observed behavior. Optical isomerism is a frequent occurrence in transition metal clusters. This phenomenon is more easily observed in the hydrogenated systems, but optical isomerism occurs in the bare transition metal clusters as well as in rare gas systems.

The growth pattern of the bare Ni clusters is nontrivial. The present study suggests that limiting the geometry optimization by assuming a best initial configuration is not recommended for transition metal clusters in this range. Like the EAM and the CEM model, a more uniform coordination of all the atoms in the cluster is observed in contrast to the noble gas systems. There are, however, differences between the lowest energy structures predicted by the present model and these alternative methods. For example for $\mathrm{Ni}_{13}$ we find the icosahedron stable but not the lowest minimum. Isomers reported by Stave and DePristo ${ }^{8}$ and found in the recent work with EAM potentials ${ }^{5}$ are also found in the present study though with different energetic ordering.

We find the Jahn-Teller effect to be important. Many instances are found, where degeneracies in the adiabatic potential surface distort the systems to lower symmetry configurations. In some cases the effect increases the topological complexity of the potential surface by adding several distinct stable distortions of the same symmetric structure. This is easily observed in $\mathrm{Ni}_{7}$ where several distinct distortions of the pentagonal bipyramid can be seen in Fig. 3. EAM and CEM methods do not appear to reproduce such distortions. In such studies, in fact, structures with high symmetry are usually reported.

Locating transition states with the present model is problematic. The crossing that takes place (accidental or by symmetry) between different adiabatic surfaces leaves cusps in the ground state PES. The popular methods ${ }^{46,54}$ used for locating transition states rely on the use of the Hessian matrix. The Hessian is numerically ill-behaved in the neighborhood of a PES orbital degeneracy. Vibronic coupling corrections need to be included in the present model if future searches for transition states are to be undertaken.

With the present model, hydrogen is found predominantly on the surface, and here a preference (by a small amount) of the bridging site (side capping) is observed over the threefold (face capping) site for the smaller clusters. The only exceptions are $\mathrm{Ni}_{3} \mathrm{H}$ and $\mathrm{Ni}_{3} \mathrm{H}_{2}$. The threefold site is favored for clusters with more than eight nickel atoms. The tetrahedral site in $\mathrm{Ni}_{4} \mathrm{H}$ and the octahedral site in $\mathrm{Ni}_{6} \mathrm{H}$ are not stable. Inspection of the electronic energy levels suggest that only distorted versions of these clusters should exist. We do not find the hydrogen inside the distorted tetrahedron or octahedron site in contrast to EAM results. ${ }^{5}$ While the Ni-H distance in these sites is larger than the $\mathrm{NiH}$ dimer equilibrium distance, the electronic energy is not sufficiently deep to stabilize the configuration. Inclusion of hydrogen inside these sites is observed in larger clusters but these constitute high energy isomers.

The $\mathrm{H}_{2}$ molecule is in all cases dissociated by the $\mathrm{Ni}$ cluster. The most favorable arrangement of the two $\mathrm{H}$ atoms on the $\mathrm{Ni}$ cluster is in two neighboring sites with a $\mathrm{Ni}$ atom bonded to both hydrogens. The addition of one or two hydrogens on the transition metal cluster frequently modifies the geometry of the $\mathrm{Ni}$ arrangement. This is to be expected from the small energy differences that exist between isomers of $\mathrm{Ni}_{n}$.

With the present model, we plan to explore further the thermodynamic properties of the systems we studied in this paper. It is also possible to study the quantum effects on the classical minima we found in this study especially in the cases where one or two hydrogens (or its isotopes) are present. Another interesting issue which will be studied in the near future is the effect that hydrogenation has on the "mobility" of the nickel substrate at finite temperatures.

\section{ACKNOWLEDGMENTS}

This work has been supported in part by the National Science Foundation under Grants Number CHE-9411000 and CHE-9625498. This research has been supported in part by the Phillips Laboratory, Air Force Material Command, USAF, through the use of the MHPCC under cooperative agreement number F29601-93-0001. The views and conclusions contained in this document are those of the authors and should not be interpreted as necessarily representing the official policies or endorsements, either expressed or implied, of Phillips Laboratory or the U.S. Government. We thank Dr. Bin Chen and Dr. Maria Gomez for helpful discussions. We also thank the referee for several careful and helpful comments.

${ }^{1}$ R. Car and M. Parrinello, Phys. Rev. Lett. 55, 2471 (1985).

${ }^{2}$ C. Z. Wang and K. M. Ho, Adv. Chem. Phys. 93, 651 (1996).

${ }^{3}$ D. R. Jennison, P. A. Schultz, and M. P. Sears, J. Chem. Phys. 106, 1856 (1997).

${ }^{4}$ B. Hartke and E. Carter, Chem. Phys. Lett. 216, 324 (1993).

${ }^{5}$ B. Chen, M. A. Gomez, M. Sehl, J. Doll, and D. L. Freeman, J. Chem. Phys. 105, 9686 (1996).

${ }^{6}$ D. L. Freeman and J. D. Doll, Annu. Rev. Phys. Chem. 47, 43 (1996).

${ }^{7}$ D. Kim, J. D. Doll, and J. E. Gubernatis, J. Chem. Phys. 106, 1641 (1997).

${ }^{8}$ M. S. Stave and A. E. Depristo, J. Chem. Phys. 97, 3386 (1992).

${ }^{9}$ T. L. Wetzel and A. E. Depristo, J. Chem. Phys. 105, 572 (1996).

${ }^{10}$ R. Hoffmann, J. Chem. Phys. 39, 1397 (1963).

${ }^{11}$ O. Sinanoğlu and K. B. Wiberg, Sigma Molecular Orbital Theory (Yale University Press, New Haven, 1970).

${ }^{12}$ A. B. Anderson, J. Chem. Phys. 64, 4046 (1976).

${ }^{13}$ M. van Schilfgaarde and W. A. Harrison, J. Phys. Chem. Solids 46, 1093 (1985).

${ }^{14}$ M. van Schilfgaarde and W. A. Harrison, Phys. Rev. B 33, 2653 (1986).

${ }^{15}$ W. A. Harrison, Phys. Rev. B 27, 3592 (1983).

${ }^{16}$ M. Menon and K. R. Subbaswamy, Phys. Rev. B 51, 17952 (1995).

${ }^{17}$ P. Ordejón, D. Lebedenko, and M. Menon, Phys. Rev. B 50, 5645 (1994).

${ }^{18}$ M. Menon and K. R. Subbaswamy, Phys. Rev. B 50, 11577 (1994).

${ }^{19}$ M. Menon and K. R. Subbaswamy, Phys. Rev. B 47, 12754 (1993).

${ }^{20}$ D. Tomańek and M. A. Schluter, Phys. Rev. B 36, 1208 (1987).

${ }^{21}$ J. N. Lathiotakis, A. N. Andriotis, M. Menon, and J. Connolly, J. Chem. Phys. 104, 992 (1996).

${ }^{22}$ M. Menon and J. Connolly, Phys. Rev. B 50, 8903 (1994). 
${ }^{23}$ N. N. Lathiotakis, A. N. Andriotis, M. Menon, and J. Connolly, Europhys. Lett. 29, 135 (1995).

${ }^{24}$ A. N. Andriotis, N. Lathiotakis, and M. Menon, Chem. Phys. Lett. 260, 15 (1996).

${ }^{25}$ A. B. Anderson and R. Hoffmann, J. Chem. Phys. 60, 4271 (1974).

${ }^{26}$ S. Lee, Annu. Rev. Phys. Chem. 47, 397 (1996).

${ }^{27}$ J. C. Slater and G. F. Koster, Phys. Rev. 94, 1498 (1954).

${ }^{28}$ L. Lian, C.-X. Su, and P. B. Armentrout, J. Chem. Phys. 96, 7542 (1992).

${ }^{29}$ E. K. Parks, L. Zhu, J. Ho, and S. J. Riley, J. Chem. Phys. 100, 7206 (1994).

${ }^{30}$ P. Mlynarski, and D. R. Salahub, J. Chem. Phys. 95, 6050 (1991).

${ }^{31}$ N. Rösch and L. Ackermann, Chem. Phys. Lett. 199, 275 (1992).

${ }^{32}$ The values of the experimental binding energy, bond length and vibrational frequency of the dimer were taken from Ref. 21. Recently, more reliable values for the binding energy and the bond length for $\mathrm{Ni}_{2}$ have been reported by Pinegar et al. ${ }^{33}$ Likewise a more accurate vibrational frequency for the dimer has been obtained by photoelectron spectroscopy of $\mathrm{Ni}_{2}^{-}$by Ho et al. ${ }^{34}$ The differences between the experimental values of the dimer that we use for the fit and those reported more recently is smaller then the discrepancy between the calculated results and experiment. Therefore, using either set of data for the fit can be expected to yield similar results.

${ }^{33}$ J. C. Pinegar, Jon D. Langenberg, C. A. Arrington, E. M. Spain, and M. D. Morse, J. Chem. Phys. 102, 666 (1995).

${ }^{34}$ J. Ho, M. L. Polak, K. M. Ervin, and W. C. Lineberger, J. Chem. Phys. 99, 8542 (1993).

${ }^{35}$ W. H. Press, S. A. Teukolsky, W. T. Vettering and B. P. Flannery, Numerical Recipes in Fortran, 2nd ed. (Cambridge University Press, Cambridge, 1992), pp. 387-448.

${ }^{36}$ J. A. Niesse and H. R. Mayne, J. Chem. Phys. 105, 4700 (1996).

${ }^{37}$ D. M. Deaven, N. Tit, J. R. Morris, and K. M. Ho, Chem. Phys. Lett. 256, 195 (1996)

${ }^{38}$ D. M. Deaven and K. M. Ho, Phys. Rev. Lett. 240, 560 (1995).
${ }^{39}$ Y. Zeiri, Phys. Rev. E 51, R2769 (1995).

${ }^{40}$ B. Hartke, J. Phys. Chem. 97, 9973 (1993).

${ }^{41}$ R. Pou-Amérigo, M. Merchán, I. Nebot-Gil, P. Malmqvist, and B. O. Roos, J. Chem. Phys. 101, 4893 (1994).

${ }^{42}$ J. A. Gray, M. L. Li, T. Nelis, and R. W. Field, J. Chem. Phys. 95, 7164 (1991).

${ }^{43}$ K. P. Huber and G. Herzberg, Molecular Structure and Molecular Spectra, IV Constants of Diatomic Molecules (Van Nostrand-Reinhold, New York, 1979).

${ }^{44}$ H. A. Jahn and E. Teller, Proc. R. Soc. London, Ser. A 161, 220 (1937).

${ }^{45}$ Z. Yu and J. Almöf, J. Chem. Phys. 95, 9167 (1991).

${ }^{46}$ C. J. Tsai and K. D. Jordan, J. Phys. Chem. 97, 11227 (1993).

${ }^{47}$ S. K. Nayak, B. Reddy, B. K. Rao, S. N. Khanna, and P. Jena, Chem. Phys. Lett. 253, 390 (1996).

${ }^{48}$ D. L. Freeman and J. D. Doll, J. Chem. Phys. 82, 462 (1985).

${ }^{49}$ The trigonal prism, the square antiprism and other simple polyhedra of interest have different binding sites for a new atom to add as a cap. In this paper we refer to a 4 capped polyhedron as a configuration where one additional atom caps the polyhedron on a 4-atom face. Equivalently a 3,4 capped polyhedron is a configuration where one additional atom caps the polyhedron on a 4-atom face and the other the 3-atom face etc. A similar notation is used in the inorganic literature. However, the 4 capped polyhedron is sometimes referred to as a square-capped configuration, while other times the symmetry of the configuration is used to distinguish it from other possible capping arrangements. This notation is useful for clusters where the atomic composition of the cluster is uniform.

${ }^{50}$ F. A. Cotton, Chemical Applications of Group Theory, 3rd ed. (Wiley, New York, 1990), p. 233.

${ }^{51}$ D. L. Freeman and J. D. Doll, Adv. Chem. Phys. 78A, 139 (1988).

${ }^{52}$ H. Yang and J. L. Whitten, J. Chem. Phys. 89, 5329 (1988).

${ }^{53}$ K. Christmann, O. Schober, G. Erlt, and M. Neumann, J. Chem. Phys. 60, 4528 (1974).

${ }^{54}$ C. J. Cerjan and W. H. Miller, J. Chem. Phys. 75, 2800 (1981). 